Article

\title{
Evaluating the Safety and Potential Risks of Food Allergy of Silk Fibroin Derived from Bombyx mori Cocoons
}

\author{
Sezin Yigit ${ }^{1}$, Nadia S. Hallaj ${ }^{1}$, James L. Sugarman ${ }^{1}$, Lester C. Chong, ${ }^{1}$ Samantha E. Roman ${ }^{1}$, Laith \\ M. Abu-Taleb ${ }^{*}$, Richard E. Goodman'2, Philip E. Johnson ${ }^{2}$, Adam M. Behrens ${ }^{1}$ \\ 1 Cambridge Crops, Inc. (d.b.a. Mori), Boston, MA 02129; laith@cambridgecrops.com \\ 2 University of Nebraska, Food Allergy Research and Research Program, Lincoln, NE, 68588-6207; \\ rgoodman2@unl.edu (R.E.G.), philip.johnson@unl.edu (P.E.J.) \\ * Corresponding author: Laith M. Abu-Taleb, laith@cambridgecrops.com; Tel.: +1 (202) 834-3174
}

\begin{abstract}
Recent studies have demonstrated silk fibroin's ability to extend the shelf life of foods by mitigating the hallmarks of spoilage, namely oxidation and dehydration. Due to the potential for this protein to become more widespread, its safety was evaluated comprehensively. First, a bacterial reverse mutation test (Ames test) was conducted in five bacterial strains. Second, an in vivo erythrocyte test was conducted with Sprague Dawley rats at doses up to $1,000 \mathrm{mg} / \mathrm{kg}$-bw $/$ day. Third, a range-finder study was conducted with Sprague Dawley rats at the highest consumption amount given solubility and oral gavage volume constrains $(500 \mathrm{mg} / \mathrm{kg}$-bw/day). Fourth, a 28 -day study in Sprague Dawley rats was conducted at the $500 \mathrm{mg} / \mathrm{kg}$-bw/day amount. Fifth, an in vitro pepsin digestion assay was performed to assess the potential for protein allergenicity. Sixth, allergenic potential was further assessed using liquid chromatography-mass spectroscopy for detection of allergenic insect proteins. Seventh, the protein sequences were subjected to bioinformatic analyses. Together, these studies raise no mutagenic, carcinogenic, toxicological, or allergenic concerns with the oral consumption of silk fibroin.
\end{abstract}

Keywords: Bombyx mori; silk fibroin; food safety; shelf-life; toxicity; mutagenicity; allergenicity

\section{Introduction}

The unique properties of silk fibroin have led to the broad investigation of its applicability and use in medical, cosmetic, and food industries. Recent developments have demonstrated the ability of silk fibroin to extend the shelf-life of various classes of foods by minimizing oxidation and dehydration $[1,2]$. The potential of this protein to reduce food waste and the corresponding likelihood of widespread consumption of silk fibroin in the context of extending the shelf-life of foods necessitates an evaluation of the potential risks.

Food waste has far-reaching socioeconomic and environmental implications that affect nations of all income levels, including food security, nutrition, and economic development. The Food and Agriculture Organization (FAO) of the United Nations estimates that over one-third of food produced globally each year is wasted, predominantly due to spoilage prior to consumption [3]. Nutrient-rich fresh foods are particularly susceptible to waste resulting from cold chain breaks, with an estimated $50 \%$ of produce and $30 \%$ of proteins wasted before reaching the end-consumer [3]. Further, the decomposition of wasted food and the subsequent energy and water inputs required for redundant production, processing, and distribution are severely detrimental to the environment.

Although the food packaging industry has shown moderate improvements over the last several decades, the cold chain and single-use plastics remain the industry standard [4-6]. Plastics demonstrate useful barrier properties for food preservation yet contribute roughly 26.8 million tons 
to landfills yearly [7]. Conversely, sustainable plastic alternatives often lack the functionality and cost-effectiveness of traditional packaging [8-11]. Recently, naturally-occurring silk proteins, comprised mostly of silk fibroin protein, demonstrated the ability to form an edible barrier on the surface of food that reduces oxidation and moisture loss, key hallmarks of spoilage [12]. As a highly tunable biomaterial with unique mechanical properties, silk fibroin presents a promising solution for sustainable shelf-life extension of a wide range of food items [1,2].

The unique properties of silk fibroin have led to the broad investigation of its applicability and use in medical, cosmetic, and food industries. Recent developments have demonstrated the ability of silk fibroin to extend the shelf-life of various classes of foods through minimizing oxidation and dehydration [1,2]. The potential of this protein to minimize food waste and the corresponding likelihood of widespread consumption of silk fibroin in the context of extending the shelf-life of foods necessitates an evaluation of the potential risks. Building upon the historical consumption of silk fibroin-containing foods (e.g. silkworms and their derivatives), this work evaluates the safety of silk fibroin using both in vitro and in vivo models [12-14].

To evaluate the potential genotoxicity of silk fibroin, an in vitro bacterial reverse mutation test (Ames test) and an in vivo mouse erythrocyte micronucleus test were performed $[15,16]$. Whole body dietary toxicity was evaluated using an in vivo 28 -day oral feeding study in Sprague Dawley rats $[17,18]$. The potential allergenicity of silk fibroin was assessed following the guidelines of the CODEX Alimentarius Commission guidance for food crops developed using biotechnology [19]. An in vitro pepsin stability study was conducted to understand the susceptibility of fibroin to digestion in pepsin [20,21]. Two pepsin:protein ratios (10:1 and 1:1) were used based on published guidelines [20,21] and recent modifications requested by some regulators. The identity of proteins in the fibroin product were determined using liquid chromatography with tandem mass spectrometry ("LC-MS/MS") with untargeted methods to determine likely protein identity and content using databases of proteins from databases of the parent organism, Bombyx mori. Additional tests were performed using LC-MS/MS to determine what proteins are in the final products. The sequences of identified proteins were compared to known allergens using bioinformatics with the AllergenOnline.org database using FASTA3 and the National Center for Biotechnology Information ("NCBI") Protein database using BLASTP, paying attention to criteria described in the CODEX Alimentarius Guidelines for food safety of food products [19]. The results of these tests along with considerations of potential uses of this product to protect foods are evaluated through the course of these studies.

\section{Materials and Methods}

\subsection{Test Substance Manufacturing \& Evaluation}

Silk fibroin protein was extracted from cut Bombyx mori ("B. mori") cocoons and manufactured as outlined in Figure 1. B. mori cocoons largely consist of two major proteins: (1) an insoluble fibroin protein and (2) a soluble sericin protein. The cocoons were first boiled in alkaline sodium carbonate solution to remove the soluble sericin. The insoluble fibroin was then removed from its container, washed in MilliQ water, and dried in room temperature conditions. The insoluble fibroin, commonly known as "degummed" fiber, was then dissolved in a highly concentrated chaotropic salt solution using a method adapted from Rockwood et al. with minimal modification [22]. The solubilized fibroin solution was then dialyzed in a water bath to remove the salt. Silk fibroin solution concentrations are determined using a bicinchoninic acid (BCA) protein assay with silk fibroin standard solutions (Advanced BioMatrix). Molecular weight of the silk fibroin solution may be determined via SDS-PAGE and subsequent Coomassie Blue staining. 


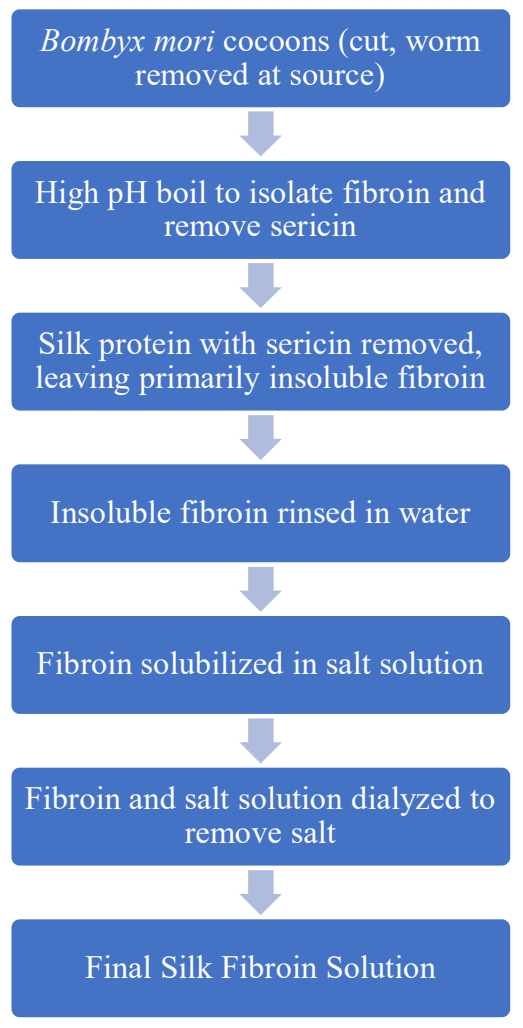

Figure 1. Silk fibroin manufacturing process overview.

\subsection{Bacterial Reverse Mutation Test (Ames Test)}

To evaluate the potential mutagenicity of silk fibroin, an Ames test was conducted at Product Safety Laboratories, Inc. (PSL; Dayton, New Jersey) using S. typhimurium strains TA1535, TA1537, TA98, TA100, and E. coli strain WP2 uvrA, per OECD guideline 471 and complying with Good Laboratory Practice (GLP) [23].

Silk fibroin was tested at doses of $31.6,100,316,1000,10,000,31,600$, and 100,000 ug/plate. This was performed in the above strains with and without pre-incubation of doses $\left(37^{\circ} \mathrm{C}\right.$ for $30 \mathrm{~min}$.) that were both metabolically inactivated and activated (via rat liver-derived S9 mix). S9 mix at 5\% v/v S9 fraction was prepared day-of and kept chilled on ice. The sterile cofactors in the S9 mix included: $8 \mathrm{mM} \mathrm{MgCl} 2,33 \mathrm{mM} \mathrm{KCl}, 100 \mathrm{mM}$ sodium phosphate buffer $\mathrm{pH}$ 7.4, $5 \mathrm{mM}$ glucose-6-phosphate and $4 \mathrm{mM}$ NADP [24]. Sterile water was used as the vehicle for silk fibroin as well as a negative control. Positive controls included the following: sodium azide $(15 \mathrm{ug} / \mathrm{mL}$ in sterile water) for S. typhimurium TA100 and TA1535, ICR 191 acridine (10 ug/mL in sterile water) for S. typhimurium TA1537, daunomycin $(60 \mathrm{ug} / \mathrm{mL}$ in sterile water) for S. typhimurium TA98, methyl methanesulfonate (25 $\mathrm{uL} / \mathrm{mL}$ in sterile water) with E. coli WP2 uvrA, and 2-aminoanthracene (100 ug/mL in DMSO) for all strains tested. All positive control materials were obtained from Molecular Toxicology, Inc. and stored refrigerated until use. Standard plate incorporation technique was used for the experiment, in which the bacterial strain, test substance or positive/negative control, and buffer with or without S9 mix were mixed and poured on agar plate. This technique was modified for strains receiving preincubated doses, in which the above mixture is incubated at $37^{\circ} \mathrm{C}$ for 30 minutes prior to plating on agar. Each test parameter was tested in triplicate. Colonies were counted with a plate counter (Colony-Doc-It $\left.{ }^{\mathrm{TM}}\right)$. The mutation factor $(\mathrm{MF})$ was calculated for each experimental group by dividing the mean revertant colony count by the mean revertant colony count for the corresponding vehicle control. A valid Ames test requires vehicle control plates to demonstrate abundant microscopic nonrevertant bacterial colony growth. Strains treated with the vehicle were to have mean revertant 
colony counts close to or within the expected laboratory historical control range and/or published values $[25,26]$. Positive controls should produce substantial increases in mean revertant colony count within the appropriate bacterial strain(s).

\subsection{In vivo Erythrocyte Micronucleus Test (Flow Cytometry)}

To evaluate potential genotoxicity of silk fibroin, an in vivo mouse erythrocyte micronucleus test was conducted at Product Safety Labs following OECD Guideline 474 and Complying with GLP. Mice for this study were supplied by Envigo Laboratories, Inc. and looked after in compliance with the latest Guide for the Care and Use of Laboratory Animals (Natl. Res. Council, 2011) [27,28]. Flow cytometry was conducted at Litron Laboratories (Rochester, NY) using their MicroFlow Basic-M Whole Blood kit.

Three groups of ten Swiss Albino (ICR) mice were used in this study. Each group consisted of five males and five females, ranging between 35-38 and 24-28 grams, respectively. Females were nulliparous and non-pregnant. Mice were selected for experimentation if they were free from clinical signs of disease or injury and had a body weight within $20 \%$ of the mean within a sex. Silk fibroin in distilled water was administered by oral gavage at a dose of $1,000 \mathrm{mg} / \mathrm{kg} / \mathrm{day}$ in a volume of $20 \mathrm{~mL} / \mathrm{kg}$ bodyweight for two days. The silk fibroin dosage was chosen as it was the maximum achievable dose level within the parameters of the test system. Distilled water served as the vehicle control $(20 \mathrm{~mL} / \mathrm{kg}$ bodyweight) and cyclophosphamide (40 mg/kg bodyweight) was the positive control. The test substance and negative control were administered on Days 1 and 2 of the study in divided doses, while the positive control was administered on Day 2 only.

Mice were group-housed in cages with solid bottoms and provided with bed-o'-cobs ${ }^{\circledR}$ bedding that was changed at least once per week. The animals were kept on a 12-hour light-dark cycle and given a six-day housing acclimation period prior to the start of testing. Throughout the duration of the study, animal room air changes were monitored 13 times per hour and the recorded temperature and relative humidity of the animal room ranged from $19-22^{\circ} \mathrm{C}$ and $50-58 \%$, respectively. The mice were supplied ad libitum with food (Teklad Global 16\% Protein Rodent Diet \# \#2016) and filtered water.

Mice were monitored for clinical observations throughout the duration of the study. Blood was collected via cardiac puncture 44-48 hours after the final dose was administered and after full anesthetization with carbon dioxide. Upon completion of sample collection, mice were euthanized via exsanguination. Flow cytometry was used to separate fluorescently-labeled erythrocytes (fluorescent labeled anti-CD71 antibody), platelets (fluorescent labeled anti-CD61 antibody), and DNA (propidium iodide, following RNAse treatment). Immature and mature erythrocytes were distinguished as CD71+/CD61- cells and CD71-/CD61- cells, respectively. A minimum target of 4,000 polychromatic erythrocytes per animal was scored for incidence of micronucleated immature erythrocytes.

Analysis of variance (ANOVA) and Bonferroni correction using GraphPad Prism (v.5.03, GraphPad Software, San Diego, CA) were used to analyze flow cytometry data. The negative control group showed micronucleated immature erythrocyte (MIE) values close to or within the expected range from method- and laboratory-specific published control data. The positive control group displayed a distinct increase in MIE values with individual and mean values outside the historical control range for negative control animals. A result was deemed positive if there was a statistically significant ( $\mathrm{p}<$ 0.05 ) increase in MIE values when compared with the negative control group in the absence of cytotoxicity. 
5 of 30

\subsection{Fourteen-Day Repeat Dose Oral Gavage Range-Finding Study}

A 14-day repeat dose study was used as a range-finder. This study was conducted at PSL according to OECD Guidelines for Testing of Chemicals, US EPA Health Effects Test Guidelines: OPPTS 870.3050, and US FDA Toxicological Principles for the Safety Assessment of Food Ingredients IV.C.4.a and was approved by the Institutional Animal Care and Use Committees (IACUC) of PSL [17,29,30]. PSL is Association for the Assessment and Accreditation of Laboratory Animal Care accredited and certified in the appropriate care of all live experimental animals and maintains current staff training, ensuring animals will be handled humanely during the experimental phase of this study in compliance with the National Research Council's 2011 Guide for the Care and Use of Laboratory Animals (8th ed.) [28].

Forty-four CRL Sprague Dawley CD IGS rats (22/sex) arrived at PSL from Charles River Laboratories, Raleigh, NC. The rats were designated by the supplier to be between six and seven weeks of age upon arrival and were acclimated for six days prior to testing. The animals were housed in a temperatureand humidity-controlled room at $19-22^{\circ} \mathrm{C}$ and 50-67\%, respectively, under a standard 12-hour lightdark cycle. Food (Envigo Teklad Gloal 16\% Protein Rodent Diet \#2016) and filtered tap water were available ad libitum, except when fasted prior to euthanasia. No known food or water contaminants were found that could potentially interfere with the results of the study. Each animal was given a sequential number in addition to being uniquely identified with a Monel self-piercing stainless-steel ear tag.

Rats (5 per sex per group, for a total of 10 per group) were administered 0,125 , 250, or $500 \mathrm{mg} / \mathrm{kg}$ bodyweight/day of silk fibroin via oral gavage. The maximum dose for this study was limited by test substance solubility in the vehicle (water) for a single gavage.

Dose administration was daily for a period of at least 14 days at approximately the same time each day ( \pm 2 hours). Individual dosages were calculated based on the most recent body weights and were adjusted each week to maintain the targeted dose level for all rats (measured in $\mathrm{mg} / \mathrm{kg}$ bodyweight/day). Animals were observed twice daily for viability. Cage-side observations were performed daily during the study. Individual body weights and food consumption were measured one day prior to study start and weekly thereafter. Food efficiency was also reported. At terminal sacrifice, all animals were weighed and euthanized by exsanguination from the abdominal aorta under isoflurane anesthesia. All animals in the study were subjected to a gross necropsy, which included examination of the external surface of the body, all orifices, musculoskeletal system, and the cranial, thoracic, abdominal, and pelvic cavities with their associated organs and tissues. All gross lesions were recorded.

\subsection{Twenty-Eight-Day Repeat-Dose Oral Toxicity Study}

A 28-day oral toxicity study was performed at PSL in accordance with the same regulations and procedures outlined in the 14-day study. Animal supplier and feed formulation were also the same.

Eighty rats (10 per sex per group, for a total of 20 per group) were administered $0,125,250$, or 500 $\mathrm{mg} / \mathrm{kg}$ bodyweight/day of silk fibroin via oral gavage. The maximum dose was determined based on the lack of adverse clinical observations when used during the 14-day study. As previously stated, the maximum dose was limited by test substance solubility in the vehicle (water) and single gavage volume.

Dose administration was daily for a period of at 28 days at approximately the same time each day $( \pm 2$ hours). Individual dosages were calculated based on the most recent body weights and were adjusted each week to maintain the targeted dose level for all rats (measured in $\mathrm{mg} / \mathrm{kg}$ bodyweight/day). 
Animals were observed twice daily for viability. Cage-side observations were performed daily during the study. Individual body weights and food consumption were measured one day prior to study start and weekly thereafter. Food efficiency was also reported. At terminal sacrifice, all animals were weighed and euthanized by exsanguination from the abdominal aorta under isoflurane anesthesia. All animals in the study were subjected to a gross necropsy, which included examination of the external surface of the body, all orifices, musculoskeletal system, and the cranial, thoracic, abdominal, and pelvic cavities with their associated organs and tissues. All gross lesions were recorded.

Selected tissues and organs were weighed wet immediately after dissection to avoid drying, including adrenals (combined), brain, epididymides (combined), kidneys (combined), liver, heart, spleen, thymus, testes (combined), uterus, ovaries without oviducts. Histological examination was performed on the preserved organs and tissues of the animals from both the control and high dose groups. The fixed tissues were trimmed, processed, embedded in paraffin, sectioned with a microtome, placed on glass microscope slides, stained with hematoxylin and eosin and examined by light microscope. Histology preparation and analysis was performed by a board-certified pathologist at Histo-Scientific Research Laboratories (HSRL).

Pathology was performed for clinical chemistry, hematology, and coagulation at necropsy. Blood was collected via the inferior vena cava: approximately $500 \mu \mathrm{L}$ of blood were collected in a pre-calibrated tube containing K2EDTA for hematology assessments. The whole blood samples were stored under refrigeration and transferred on cold packs. Hematology included erythrocyte count (RBC), hemoglobin concentration (HGB), hematocrit (HCT), mean corpuscular volume (MCV), mean corpuscular hemoglobin $(\mathrm{MCH})$, red cell distribution width (RDW), absolute reticulocyte count (ARET), platelet count (PLT), total white blood cell (WBC) and differential leukocyte count; mean corpuscular hemoglobin concentration (MCHC) was also calculated. Separate blood smears, stained with New Methylene Blue or Wright-Giemsa stain, were prepared from each animal to substantiate hematology findings. Approximately $1.8 \mathrm{~mL}$ of blood was collected in a pre-calibrated tube containing $3.2 \%$ sodium citrate for coagulation analysis. These samples were centrifuged and the plasma stored in a $-80^{\circ} \mathrm{C}$ freezer until analysis. Coagulation included prothrombin time (PT) and activated partial thromboplastin time (APTT). Approximately $1000 \mu \mathrm{L}$ of blood were collected in a tube containing no preservative for clinical chemistry assessments. These samples were centrifuged, and the serum stored in a $-80^{\circ} \mathrm{C}$ freezer until analysis. Clinical chemistry included serum aspartate aminotransferase (AST), serum alanine aminotransferase (ALT), sorbitol dehydrogenase (SDH), alkaline phosphatase (ALKP), total bilirubin (TBIL), urea nitrogen (BUN), blood creatinine (CREAT), total cholesterol (CHOL), triglycerides (TRIG), fasting glucose (GLUC), total serum protein (TP), albumin (ALB), globulin (GLOB), calcium (CA), inorganic phosphorus (PHOS), sodium (NA), potassium $(\mathrm{K})$, chloride $(\mathrm{CL})$.

Animals were fasted overnight prior to urine collection and samples were refrigerated until analysis. Urinalysis included quality (QUAL), pH, ketone (UKET), color (UCOL), glucose (UGLU), bilirubin (UBIL), clarity (UCLAR), specific gravity (SG), blood (UBLO), volume (UVOL), protein (UPRO), urobilinogen (URO) and microscopic urine sediment examination.

\subsection{Digestion \& Allergenicity Studies}

\subsubsection{Pepsin Digestion}

To determine whether silk fibroin contains digestion-resistant polypeptides that might elicit an allergenic response, an in vitro digestion assay at $\mathrm{pH} 2$ in simulated gastric fluid (SGF) at

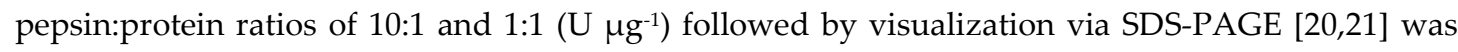
performed. Stock solutions of pepsin were prepared at $1.05 \times 106$ units/mL (for 10:1) and 1.05x105 units/mL (for 1:1) in simulated gastric fluid (SGF), respectively. A silk fibroin stock was prepared 
$42 \mathrm{mg} / \mathrm{mL}$ in sterile water. To begin digestion, $1.43 \mathrm{~mL}(60 \mathrm{mg})$ of silk fibroin stock was combined with $570 \mu \mathrm{L}$ of the relevant pepsin stock and placed in a pre-heated incubator set to $37^{\circ} \mathrm{C}$ and 120 RPM. Samples of $0.2 \mathrm{~mL}$ were taken at $0,2,5,10,20,30$, and 60 minutes. Controls to assess pepsin auto-digestion (pepsin without silk fibroin) and the thermal stability of the test substance (silk fibroin without pepsin) were tested at 0 and 60 minutes. All samples were collected in vials containing $70 \mu \mathrm{L}$ of $0.7 \mathrm{M}$ sodium carbonate and $70 \mu \mathrm{L}$ of $5 \mathrm{x}$ Laemmli loading buffer. Samples were vortexed and heated at a temperature greater than $80^{\circ} \mathrm{C}$ for 10 minutes to inactivate pepsin prior to electrophoresis at $350 \mathrm{ug} /$ well. After electrophoresis, the gels were stained using Coomassie Blue for visualization. To assess the extent of digestion, a reference gel with wells containing $350 \mu \mathrm{g}$ silk fibroin (corresponding to $0 \%$ digestion), $175 \mu \mathrm{g}$ (50\% digestion), $87.5 \mu \mathrm{g}$ ( $75 \%$ digestion), $43.8 \mu \mathrm{g}(87.5 \%$ digestion), $21.9 \mu \mathrm{g}$ (93.7\% digestion), $10.9 \mu \mathrm{g}$ (96.9\% digestion), $5.5 \mu \mathrm{g}(98.4 \%)$, and $2.7 \mu \mathrm{g}(99.2 \%)$ was run. As a positive control, bovine serum albumin (BSA) was subjected to digestion by pepsin in SGF at the same ratios as silk fibroin, 10:1 and 1:1.

\subsubsection{Identification \& Evaluation of Potential Allergens}

Four samples, representative of silk fibroin and potential manufacturing contaminants, were evaluated for allergenic potential. These samples included the B. mori pupa, cocoon, degummed silk fibroin, and silk fibroin powder. Samples were sent to the Harvard Center for Mass Spectrometry Proteomics Laboratory for sample preparation and analysis via mass spectroscopy (MS). To prepare cocoon, degummed silk fibroin, and silk fibroin powder product samples, $20 \mu \mathrm{L}$ of dialyzed material at $(14 \mathrm{mg} / \mathrm{ml}$, for a total of approximately $280 \mu \mathrm{g}$ of protein) was reduced, alkylated, and digested by trypsin using a FASP protocol. Samples were dried and resuspended in $30 \mu \mathrm{L}$ of $0.1 \%$ trifluoracetic acid (TFA). $5 \mathrm{~mL}$ of this solution was injected for MS analysis (Orbitrap Elite Hybrid Ion TrapOrbitrap Mass Spectrometer using a WATERS Aquity \#186008795 nanoEase M/Z Peptide BEH column). The B. mori pupa sample was prepared by combining Covaris DF buffer (containing urea) with the pupa sample (sample $1=7.6 \mathrm{mg}$, sample $2=6.62 \mathrm{mg}$ ) to give a $1 \% \mathrm{w} / \mathrm{v}$ solution, and then sonicated using a Covaris S220 shearing device. Samples $(1=76 \mu \mathrm{g}, 2=66 \mu \mathrm{g})$ of the resulting extract were used in FASP and analyzed as described above. An estimated $2.5 \mu \mathrm{g}$ of pupa material was injected.

The raw mass spectrometry data (Thermo.raw files) used for analysis in this experiment were provided by the Harvard Center for Mass Spectrometry Proteomics Laboratory. Two separate sets of MS analysis were performed in separate experiments. In total, there are 3 MS runs of pupa material, 1 run of cocoon material, 1 run of degummed fibroin product and 3 runs of fibroin powder product.

Data were analyzed using PEAKSQ version 8.5. Full settings used are available upon request. Data were taken directly from PEAKS and label-free data was exported for analysis in MS Excel. This study considers only data relevant to designated proteins of interest, as outlined below.

The $-10 \log \mathrm{P}$ score is a measure of protein identification used by PEAKS software. The P-value is converted to $-10^{*} \log 10$ (P-value) for ease of understanding. A more significant match will have a higher $-10 \lg \mathrm{P}$ value. Additionally, a $\mathrm{P}$-value of $1 \%$ is equivalent to $-10 \lg \mathrm{P}$ of 20 . This score is dependent upon the database used as well as the number and quality of peptide spectra. The number of peptides identified for each protein sequence is given here as an additional measure of identification quality. Human Proteome Organization (HUPO)-Proteomics Standards Initiative (PSI) demand at least two unique peptides for a protein identification.

\subsubsection{Bioinformatics \& Literature Searches}

Literature searches and bioinformatics evaluations for possible risks of allergy were performed using the amino acid sequences of the identified, intended proteins that represent fibroin, and proteins that have been suggested as possible allergens from the adult insect or pupae. Bioinformatics methods 
include Full-length FASTA3, sliding 80mer window FASTA3 and eight-amino acid identity matches using AllergenOnline.org, version 20 as described for transgenic proteins in bacterial wilt-resistant bananas [31]. The primary concern for allergy is whether the proteins are known to be allergens or are sufficiently sequence identical to an allergen to suspect possible IgE cross-reactivity. Additional searches were performed with BLASTP using the NCBI Protein database to confirm those findings.

\section{Results}

\subsection{Bacterial Reverse Mutation Test (Ames Test)}

Results are indicative of mutagenicity if three criteria were met: (1) a substantial increase in revertant colony counts as defined by MF $>2$ for bacterial strains TA98, TA100, and WP2 uvrA and MF $>3$ for bacterial strains TA1535 and TA1537; (2) the mean MF value(s) must lie outside the historical laboratory control range; and (3) the increase in revertant colony counts is dose related and/or reproducible, where increases are obtained at more than one experimental point.

The mean, standard deviation, and mutation factor for each set of triplicate plates are found in Tables $1 \mathrm{~A}-1 \mathrm{E}$. In the case of each strain treated with the vehicle, the mean revertant colony counts were close to or within the expected range, considering the laboratory historical control range and/or published values in Table S1 of the Supplemental Information [25]. In each phase of the test, the positive control substances caused the expected substantial increases in revertant colony counts in both the absence and presence of $\mathrm{S} 9 \mathrm{mix}$. The validity of the test is demonstrated through this confirmation of both the sensitivity of the test and the activity of the S9 mix.

Table 1A. Mean revertant colony counts of bacterial strain TA1535 \pm SD (Ames test)

\begin{tabular}{|c|c|c|c|c|c|c|c|c|c|}
\hline \multirow[b]{3}{*}{ Treatment } & \multirow[b]{3}{*}{$\begin{array}{c}\text { Dose, } \\
\mu \mathrm{g} / \text { plate }\end{array}$} & \multicolumn{4}{|c|}{ Plate Incorporation Method } & \multicolumn{4}{|c|}{ Pre-Incubation Method } \\
\hline & & \multicolumn{2}{|c|}{-59} & \multicolumn{2}{|c|}{ +S9 } & \multicolumn{2}{|c|}{-59} & \multicolumn{2}{|c|}{ +S9 } \\
\hline & & Count & MF & Count & MF & Count & MF & Count & MF \\
\hline Sterile Water & N/A & $12 \pm 6.1$ & 1.00 & $9 \pm 2.3$ & 1.00 & $12 \pm 5.3$ & 1.00 & $13 \pm 3.8$ & 1.00 \\
\hline Silk Fibroin & 31.6 & $13 \pm 1.2$ & 1.08 & $13 \pm 4.9$ & 1.44 & $14 \pm 2.6$ & 1.17 & $10 \pm 2.6$ & 0.77 \\
\hline Silk Fibroin & 100 & $13 \pm 2.0$ & 1.08 & $9 \pm 5.5$ & 1.00 & $9 \pm 3.5$ & 0.75 & $9 \pm 0.6$ & 0.69 \\
\hline Silk Fibroin & 316 & $12 \pm 1.5$ & 1.0 & $13 \pm 1.0$ & 1.44 & $11 \pm 2.1$ & 0.92 & $12 \pm 0.6$ & 0.92 \\
\hline Silk Fibroin & 1000 & $15 \pm 1.5$ & 1.25 & $11 \pm 3.2$ & 1.22 & $12 \pm 2.5$ & 1.00 & $12 \pm 2.9$ & 0.92 \\
\hline Silk Fibroin & 3160 & $12 \pm 0.6$ & 1.00 & $12 \pm 4.2$ & 1.33 & $16 \pm 2.9$ & 1.33 & $8 \pm 0.6$ & 0.62 \\
\hline Silk Fibroin & 10000 & $15 \pm 7.0$ & 1.25 & $10 \pm 3.2$ & 1.11 & $13 \pm 1.5$ & 1.08 & $12 \pm 2.9$ & 0.92 \\
\hline Silk Fibroin & 31600 & $10 \pm 1.2$ & 0.83 & $16 \pm 2.1$ & 1.78 & $15 \pm 7.8$ & 1.25 & $11 \pm 5.2$ & 0.85 \\
\hline Silk Fibroin & 100000 & $17 \pm 4.5$ & 1.42 & $10 \pm 4.5$ & 1.11 & $11 \pm 2.1$ & 0.92 & $15 \pm 3.2$ & 1.15 \\
\hline Sodium Azide & 1.5 & $807 \pm 30.4$ & 67.25 & - & - & $805 \pm 11.7$ & 67.08 & - & - \\
\hline $2-\mathrm{AA}$ & 10 & - & - & $412 \pm 44.5$ & 45.78 & - & - & $347 \pm 19.2$ & 26.69 \\
\hline
\end{tabular}

$\mathrm{n}=3$; $\mathrm{SD}$, standard deviation; count, revertant colony counts per plate; $\mathrm{MF}$, mutation factor; ${ }^{\mathrm{S}} \mathrm{S}$, with/without activation factor S9; 2-AA, 2-aminoanthracene

$\mathrm{U} 2$ replicates due to $\mathrm{U}$ - contamination, plate unreadable 
9 of 30

Table 1B. Mean revertant colony counts of bacterial strain TA1537 \pm SD (Ames test)

\begin{tabular}{|c|c|c|c|c|c|c|c|c|c|}
\hline & & \multicolumn{4}{|c|}{ Plate Incorporation Method } & \multicolumn{4}{|c|}{ Pre-Incubation Method } \\
\hline & & \multicolumn{2}{|c|}{-59} & \multicolumn{2}{|c|}{ +S9 } & \multicolumn{2}{|c|}{$-S 9$} & \multicolumn{2}{|c|}{$+\mathrm{S} 9$} \\
\hline Treatment & $\begin{array}{c}\text { Dose, } \\
\mu g / \text { plate }\end{array}$ & Count & MF & Count & MF & Count & MF & Count & MF \\
\hline Sterile Water & N/A & $12 \pm 2.1$ & 1.00 & $14 \pm 3.5$ & 1.00 & $16 \pm 3.5$ & 1.00 & $14 \pm 4.6$ & 1.00 \\
\hline Silk Fibroin & 31.6 & $15 \pm 3.6$ & 1.25 & $16 \pm 2.3$ & 1.14 & $15 \pm 4.0$ & 0.94 & $11 \pm 2.3$ & 0.79 \\
\hline Silk Fibroin & 100 & $13 \pm 5.1$ & 1.08 & $14 \pm 3.5$ & 1.00 & $11 \pm 1.2$ & 0.69 & $12 \pm 2.1$ & 0.86 \\
\hline Silk Fibroin & 316 & $12 \pm 2.3$ & 1.00 & $16 \pm 2.9$ & 1.14 & $14 \pm 3.8$ & 0.88 & $15 \pm 2.5$ & 1.07 \\
\hline Silk Fibroin & 1000 & $7 \pm 2.3$ & 0.58 & $12 \pm 1.7$ & 0.86 & $18 \pm 7.5$ & 1.13 & $17 \pm 1.4 \mathrm{U}$ & 1.21 \\
\hline Silk Fibroin & 3160 & $10 \pm 4.7$ & 0.83 & $14 \pm 1.5$ & 1.00 & $12 \pm 0.6$ & 0.75 & $17 \pm 4.2$ & 1.21 \\
\hline Silk Fibroin & 10000 & $15 \pm 2.6$ & 1.25 & $13 \pm 4.0$ & 0.93 & $17 \pm 2.3$ & 1.06 & $18 \pm 3.2$ & 1.29 \\
\hline Silk Fibroin & 31600 & $16 \pm 3.0$ & 1.33 & $13 \pm 3.8$ & 0.93 & $18 \pm 4.7$ & 1.13 & $15 \pm 5.1$ & 1.07 \\
\hline Silk Fibroin & 100000 & $20 \pm 2.9$ & 1.67 & $21 \pm 4.0$ & 1.50 & $18 \pm 4.0$ & 1.13 & $14 \pm 2.5$ & 1.00 \\
\hline ICR191 acridine & 1.5 & $287 \pm 52.7$ & 23.92 & - & - & $5948 \pm 379.9$ & 371.75 & - & - \\
\hline 2-AA & 10 & - & - & $243 \pm 85.9$ & 17.36 & - & - & $233 \pm 37.2$ & - \\
\hline
\end{tabular}

$\mathrm{n}=3$; SD, standard deviation; count, revertant colony counts per plate; $\mathrm{MF}$, mutation factor; $\pm S 9$, with/without activation factor S9; 2-AA, 2-aminoanthracene

$\mathrm{U} 2$ replicates due to $\mathrm{U}$ - contamination, plate unreadable

Table 1C. Mean revertant colony counts of bacterial strain TA98 \pm SD (Ames test)

\begin{tabular}{|c|c|c|c|c|c|c|c|c|c|}
\hline \multirow[b]{3}{*}{ Treatment } & \multirow{3}{*}{$\begin{array}{c}\text { Dose, } \\
\mu \mathrm{g} / \text { plate }\end{array}$} & \multicolumn{4}{|c|}{ Plate Incorporation Method } & \multicolumn{4}{|c|}{ Pre-Incubation Method } \\
\hline & & \multicolumn{2}{|c|}{-59} & \multicolumn{2}{|l|}{$+\mathrm{S} 9$} & \multicolumn{2}{|c|}{$-S 9$} & \multicolumn{2}{|c|}{$+S 9$} \\
\hline & & Count & MF & Count & MF & Count & MF & Count & MF \\
\hline Sterile Water & N/A & $24 \pm 1.2$ & 1.00 & $28 \pm 4.9$ & 1.00 & $21 \pm 1.2$ & 1.00 & $27 \pm 4.9$ & 1.00 \\
\hline Test Substance & 31.6 & $23 \pm 2.5$ & 0.96 & $23 \pm 1.2$ & 0.82 & $23 \pm 3.8$ & 1.10 & $25 \pm 1.5$ & 0.93 \\
\hline Test Substance & 100 & $22 \pm 4.0$ & 0.92 & $27 \pm 5.6$ & 0.96 & $20 \pm 5.1$ & 0.95 & $26 \pm 3.1$ & 0.96 \\
\hline Test Substance & 316 & $22 \pm 2.6$ & 0.92 & $29 \pm 5.5$ & 1.04 & $23 \pm 2.3$ & 1.10 & $23 \pm 3.1$ & 0.85 \\
\hline Test Substance & 1000 & $24 \pm 10.0$ & 1.00 & $29 \pm 4.0$ & 1.04 & $22 \pm 3.5$ & 1.05 & $24 \pm 5.7$ & 0.89 \\
\hline Test Substance & 3160 & $26 \pm 3.6$ & 1.08 & $25 \pm 5.7$ & 0.89 & $31 \pm 3.8$ & 1.48 & $27 \pm 2.6$ & 1.00 \\
\hline Test Substance & 10000 & $20 \pm 4.5$ & 0.83 & $28 \pm 3.1$ & 1.00 & $21 \pm 6.9$ & 1.00 & $34 \pm 3.5$ & 1.26 \\
\hline Test Substance & 31600 & $21 \pm 4.2$ & 0.88 & $23 \pm 2.3$ & 0.82 & $23 \pm 1.5$ & 1.10 & $24 \pm 2.1$ & 0.89 \\
\hline Test Substance & 100000 & $26 \pm 4.0$ & 1.08 & $29 \pm 5.9$ & 1.04 & $21 \pm 1.5$ & 1.00 & $25 \pm 4.0$ & 0.93 \\
\hline Daunomycin & 1.5 & $1346 \pm 63.4$ & 56.08 & - & - & $876 \pm 14.6$ & 41.71 & - & - \\
\hline $2-\mathrm{AA}$ & 10 & - & - & $3205 \pm 300.9$ & 114.46 & - & - & $3512 \pm 354.0$ & 130.07 \\
\hline
\end{tabular}

$\mathrm{n}=3$; SD, standard deviation; count, revertant colony counts per plate; $\mathrm{MF}$, mutation factor; \pm S9, with/without activation factor S9; 2-AA, 2-aminoanthracene 
Table 1D. Mean revertant colony counts of bacterial strain TA100 \pm SD (Ames test)

\begin{tabular}{|c|c|c|c|c|c|c|c|c|c|}
\hline \multirow[b]{3}{*}{ Treatment } & \multirow[b]{3}{*}{$\begin{array}{c}\text { Dose, } \\
\mu \mathrm{g} / \text { plate }\end{array}$} & \multicolumn{4}{|c|}{ Plate Incorporation Method } & \multicolumn{4}{|c|}{ Pre-Incubation Method } \\
\hline & & \multicolumn{2}{|c|}{$-S 9$} & \multicolumn{2}{|c|}{ +S9 } & \multicolumn{2}{|c|}{$-S 9$} & \multicolumn{2}{|l|}{ +S9 } \\
\hline & & Count & MF & Count & MF & Count & MF & Count & MF \\
\hline Sterile Water & $\mathrm{N} / \mathrm{A}$ & $89 \pm 8.1$ & 1.00 & $110 \pm 5.6$ & 1.00 & $115 \pm 27.2$ & 1.00 & $121 \pm 27.0$ & 1.00 \\
\hline Test Substance & 31.6 & $122 \pm 8.4$ & 1.37 & $113 \pm 13.3$ & 1.03 & $91 \pm 15.5$ & 0.79 & $122 \pm 10.0$ & 1.01 \\
\hline Test Substance & 100 & $101 \pm 16.6$ & 1.13 & $117 \pm 7.0$ & 1.06 & $99 \pm 4.6$ & 0.86 & $126 \pm 7.9$ & 1.04 \\
\hline Test Substance & 316 & $112 \pm 6.5$ & 1.26 & $106 \pm 11.1$ & 0.96 & $107 \pm 17.6$ & 0.93 & $133 \pm 13.1$ & 1.10 \\
\hline Test Substance & 1000 & $95 \pm 10.5$ & 1.07 & $117 \pm 10.1$ & 1.06 & $124 \pm 2.1$ & 1.08 & $113 \pm 16.6$ & 0.93 \\
\hline Test Substance & 3160 & $107 \pm 3.6$ & 1.20 & $102 \pm 7.2$ & 0.93 & $110 \pm 12.6$ & 0.96 & $111 \pm 2.0$ & 0.92 \\
\hline Test Substance & 10000 & $112 \pm 11.8$ & 1.26 & $122 \pm 16.4$ & 1.11 & $116 \pm 9.5$ & 1.01 & $115 \pm 3.0$ & 0.95 \\
\hline Test Substance & 31600 & $107 \pm 15.0$ & 1.20 & $117 \pm 16.5$ & 1.06 & $103 \pm 9.5$ & 0.90 & $104 \pm 11.9$ & 0.86 \\
\hline Test Substance & 100000 & $105 \pm 10.0$ & 1.18 & $124 \pm 16.5$ & 1.13 & $105 \pm 8.7$ & 0.91 & $108 \pm 12.9$ & 0.89 \\
\hline Sodium azide & 1.5 & $696 \pm 36.1$ & 7.82 & - & - & $476 \pm 37.2$ & 4.14 & - & - \\
\hline $2-\mathrm{AA}$ & 10 & - & - & $3812 \pm 184.2$ & 34.65 & - & - & $2708 \pm 245.0$ & 22.38 \\
\hline
\end{tabular}

$\mathrm{n}=3$; SD, standard deviation; count, revertant colony counts per plate; MF, mutation factor; $\pm S 9$, with/without activation factor S9; 2-AA, 2-aminoanthracene

Table 1E. Mean revertant colony counts of bacterial strain EC WP2 uvrA \pm SD (Ames test)

\begin{tabular}{|c|c|c|c|c|c|c|c|c|c|}
\hline \multirow[b]{3}{*}{ Treatment } & \multirow{3}{*}{$\begin{array}{c}\text { Dose, } \\
\mu \mathrm{g} / \text { plate }\end{array}$} & \multicolumn{4}{|c|}{ Plate Incorporation Method } & \multicolumn{4}{|c|}{ Pre-Incubation Method } \\
\hline & & \multicolumn{2}{|c|}{-59} & \multicolumn{2}{|c|}{$+S 9$} & \multicolumn{2}{|c|}{$-S 9$} & \multicolumn{2}{|c|}{$+S 9$} \\
\hline & & Count & MF & Count & MF & Count & MF & Count & MF \\
\hline Sterile Water & $\mathrm{N} / \mathrm{A}$ & $38 \pm 3.1^{C}$ & 1.00 & $45 \pm 5.9$ & 1.00 & $38 \pm 4.0$ & 1.00 & $42 \pm 2.1$ & 1.00 \\
\hline Test Substance & 31.6 & $38 \pm 3.5$ & 1.00 & $43 \pm 3.2$ & 0.96 & $44 \pm 2.9$ & 1.16 & $49 \pm 2.5$ & 1.17 \\
\hline Test Substance & 100 & $35 \pm 4.7$ & 0.92 & $39 \pm 7.8$ & 0.87 & $38 \pm 2.1$ & 1.00 & $46 \pm 4.9$ & 1.10 \\
\hline Test Substance & 316 & $41 \pm 4.4$ & 1.08 & $43 \pm 2.6$ & 0.96 & $34 \pm 5.6$ & 0.89 & $45 \pm 8.3$ & 1.07 \\
\hline Test Substance & 1000 & $44 \pm 7.5$ & 1.16 & $43 \pm 6.5$ & 0.96 & $32 \pm 4.7$ & 0.84 & $40 \pm 2.3$ & 0.95 \\
\hline Test Substance & 3160 & $37 \pm 6.6$ & 0.97 & $37 \pm 6.7$ & 0.82 & $37 \pm 4.2$ & 0.97 & $39 \pm 5.3$ & 0.93 \\
\hline Test Substance & 10000 & $35 \pm 6.8$ & 0.92 & $50 \pm 6.5$ & 1.11 & $35 \pm 5.0$ & 0.92 & $52 \pm 6.1$ & 1.24 \\
\hline Test Substance & 31600 & $35 \pm 10.8$ & 0.92 & $41 \pm 2.6$ & 0.91 & $37 \pm 7.0$ & 0.97 & $41 \pm 6.8$ & 0.98 \\
\hline Test Substance & 100000 & $41 \pm 6.1$ & 1.08 & $48 \pm 3.0$ & 1.07 & $36 \pm 2.1$ & 0.95 & $44 \pm 2.5$ & 1.05 \\
\hline MMS & 1.5 & $881 \pm 58.0$ & 23.18 & - & - & $457 \pm 3.5$ & 12.03 & - & - \\
\hline $2-\mathrm{AA}$ & 10 & - & - & $117 \pm 2.5$ & 2.60 & - & - & $111 \pm 8.9$ & 2.64 \\
\hline
\end{tabular}

$\mathrm{n}=3$; SD, standard deviation; count, revertant colony counts per plate; MF, mutation factor; $\pm S 9$, with/without activation factor S9; MMS, methyl methanesulfonate; 2-AA, 2-aminoanthracene C - contamination, did not obscure count 
Contamination that did not obscure mean revertant colony counts was noted on a singular plate in the vehicle control for the E. coli strain in the plate incorporation method. Contamination that did obscure plate counts was noted in two individual plates: strain TA1535 at a dose of $3160 \mu \mathrm{g} / \mathrm{plate}$ using the standard incorporation method and strain TA1537 at a dose of $1000 \mu \mathrm{g} /$ plate using the preincubation method. None of the three individual contaminated plates impacted mutagenicity evaluation in this study as they did not meet the predefined criteria for a positive result. Additionally, no signs of precipitation or toxicity were noted in any of the strains.

There were no concentration-related or substantial test substance-related increases in the number of revertant colonies observed with strains TA1535, TA1537, TA98, TA100 or E. Coli WP2 uvrA in both the absence and presence of $\mathrm{S} 9$ using either the plate incorporation or the pre-incubation method.

Based on these results, silk fibroin did not elicit any evidence of bacterial mutagenicity in the Bacterial Reverse Mutation (Ames) Test.

\subsection{In Vivo Mouse Erythrocyte Micronucleus Test (Flow Cytometry)}

Mean animal body weights (Table 2) and cage-side observations, a summary of average percent micronucleated reticulocytes (\% MN-RET), percent of reticulocytes (\% RET), and percent micronucleated normochromatic erythrocytes (\% MN-NCE) (Table 3A-3C), dot plots of representative blood samples for both male and female mice (Figures 2A and 2B, respectively), and relevant historical control data (Supplemental Information Tables S2 and S3) [32-34]. The negative control group showed micronucleated immature erythrocyte values consistent with PSL historical data and the positive control caused a statistically significant increase in MIE with individual and mean values consistent with PSL historical data and outside of the historical control range for negative control animals. All results were within the expected expected ranges for both the positive and negative controls.

Table 2. Mean body weight \pm SD, g (mouse erythrocyte micronucleus test)

\begin{tabular}{ccc}
\hline Group & $\mathrm{M}$ & $\mathrm{F}$ \\
\hline $\begin{array}{c}\text { Vehicle control, } 0 \\
\mathrm{mg} / \mathrm{kg} / \text { day }\end{array}$ & $37.0 \pm 1.0$ & $26.0 \pm 0.7$ \\
Test substance, 1000 & $37.0 \pm 0.7$ & $27.0 \pm 1.0$ \\
$\mathrm{mg} / \mathrm{kg} /$ day & & \\
Positive control, 40 & $36.0 \pm 1.0$ & $26.2 \pm 1.5$ \\
$\mathrm{mg} / \mathrm{kg} /$ day & & \\
\hline
\end{tabular}

$\mathrm{n}=5$; SD, standard deviation; SEM, standard error of mean; M, male; $\mathrm{F}$, female

Table 3A. Summary of average percent micronucleated reticulocytes (\% MN-RET)

\begin{tabular}{lcccc}
\hline \% MN-RET & \multicolumn{2}{c}{ Mean \pm SEM } & M & -value \\
\hline Group & $\mathrm{M}$ & $\mathrm{F}$ & - & $\mathrm{F}$ \\
\hline Vehicle control, $0 \mathrm{mg} / \mathrm{kg} /$ day & $0.12 \pm 0.01$ & $0.11 \pm 0.01$ & & $\mathrm{~ns}$ \\
Test substance, 1000 & & $\mathrm{~ns}$ & $p<0.001$ \\
mg/kg/day & $0.12 \pm 0.02$ & $0.14 \pm 0.01$ & & $p<0.001$ \\
Positive control, 40 & & & \\
mg/kg/day & $2.06 \pm 0.35$ & $1.41 \pm 0.15$ & & \\
\hline
\end{tabular}

$\mathrm{n}=5 ; \mathrm{ns}$, not significant $(p>0.05)$ 
\% MN-RET, frequency (\%) of positive CD71 micronucleated reticulocytes (i.e., micronucleated immature erythrocytes [MIEs]); SEM, standard error of mean; M, male; F, female

Table 3B. Percent of reticulocytes (\% RET)

\begin{tabular}{|c|c|c|c|c|c|}
\hline \multirow{2}{*}{$\begin{array}{c}\% \text { RET } \\
\text { Group }\end{array}$} & & \multicolumn{2}{|c|}{ Mean \pm SEM } & \multicolumn{2}{|c|}{$p$-value } \\
\hline & & M & $\mathrm{F}$ & M & F \\
\hline $\begin{array}{l}\text { Vehicle control, } \\
\mathrm{mg} / \mathrm{kg} / \text { day }\end{array}$ & 0 & $1.88 \pm 0.12$ & $1.52 \pm 0.10$ & - & - \\
\hline $\begin{array}{l}\text { Test substance, } \\
\mathrm{mg} / \mathrm{kg} / \text { day }\end{array}$ & 1000 & $1.55 \pm 0.14$ & $1.42 \pm 0.21$ & ns & ns \\
\hline $\begin{array}{l}\text { Positive control, } \\
\mathrm{mg} / \mathrm{kg} / \text { day }\end{array}$ & 40 & $0.62 \pm 0.08$ & $0.42 \pm 0.08$ & $p<0.001$ & $p<0.001$ \\
\hline
\end{tabular}

$\mathrm{n}=5 ; \mathrm{ns}$, not significant $(p>0.05)$

$\%$ RET, frequency (\%) of CD71 positive reticulocytes; SEM, standard error of mean; $\mathrm{M}$, male; F, female

Table 3C. Percent micronucleated normochromatic erythrocytes (\% MN-NCE)

\begin{tabular}{|c|c|c|c|c|}
\hline \multirow{2}{*}{$\begin{array}{l}\% \text { MN-NCE } \\
\text { Group }\end{array}$} & \multicolumn{2}{|c|}{ Mean \pm SEM } & \multicolumn{2}{|c|}{$p$-value } \\
\hline & M & $\mathrm{F}$ & M & $\mathrm{F}$ \\
\hline $\begin{array}{l}\text { Vehicle control, } 0 \\
\mathrm{mg} / \mathrm{kg} / \text { day }\end{array}$ & $0.11 \pm 0.01$ & $0.09 \pm 0.01$ & - & - \\
\hline $\begin{array}{l}\text { Test substance, } 1000 \\
\mathrm{mg} / \mathrm{kg} / \text { day }\end{array}$ & $0.11 \pm 0.01$ & $0.13 \pm 0.01$ & ns & $\mathrm{ns}$ \\
\hline $\begin{array}{l}\text { Positive control, } 40 \\
\mathrm{mg} / \mathrm{kg} / \text { day }\end{array}$ & $0.14 \pm 0.02$ & $0.13 \pm 0.01$ & ns & ns \\
\hline$n=5 ; n s$, not signi & 05) & & & \\
\hline
\end{tabular}



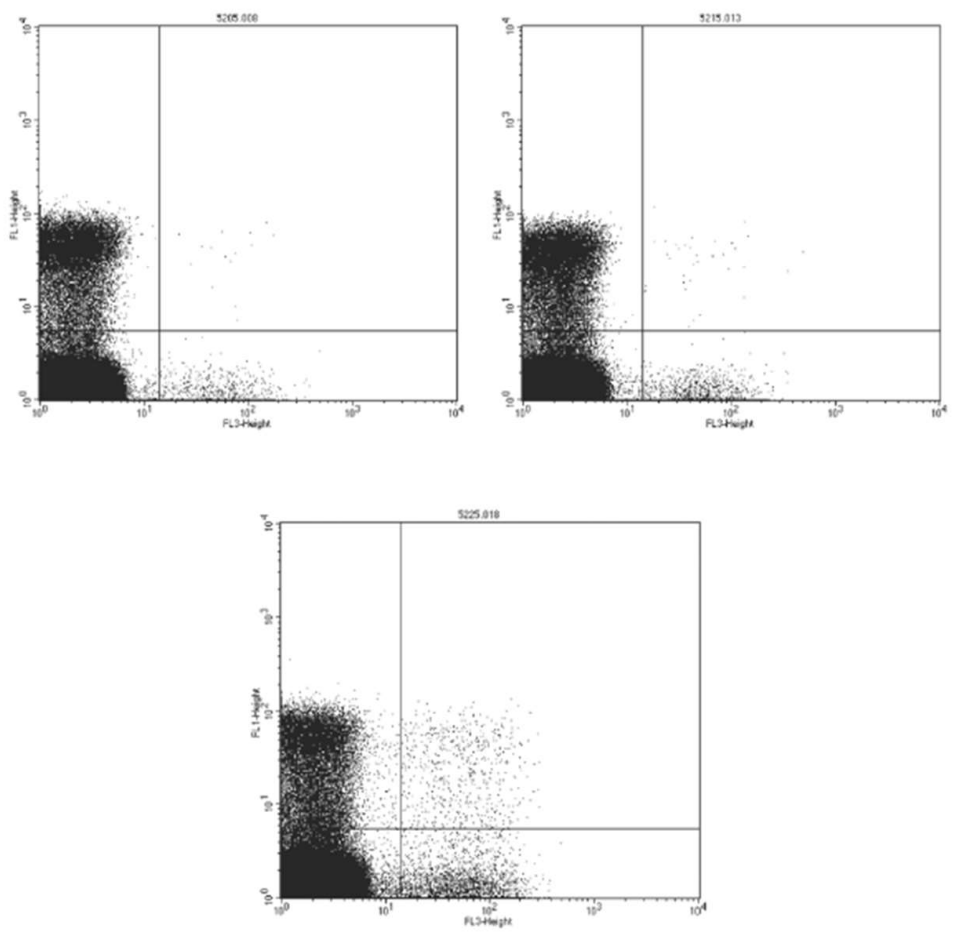

Figure 2A. Three Representative Bivariates (Male). These representative bivariates of test facility submitted Samples 5205, 5215, and 5225 illustrate the resolution of the various erythrocyte populations in mouse peripheral blood: Lower Left quadrant $=$ NCE [cells which are low in green and red fluorescence]; Lower Right $=$ MN-NCE [cells high in red (PI) fluorescence]; Upper Left = CD71 positive RET [cells with green (CD71) fluorescence]; Upper Right = CD71 positive MN-RET [cells with both red and green fluorescence; the population of primary interest for this analysis]. 

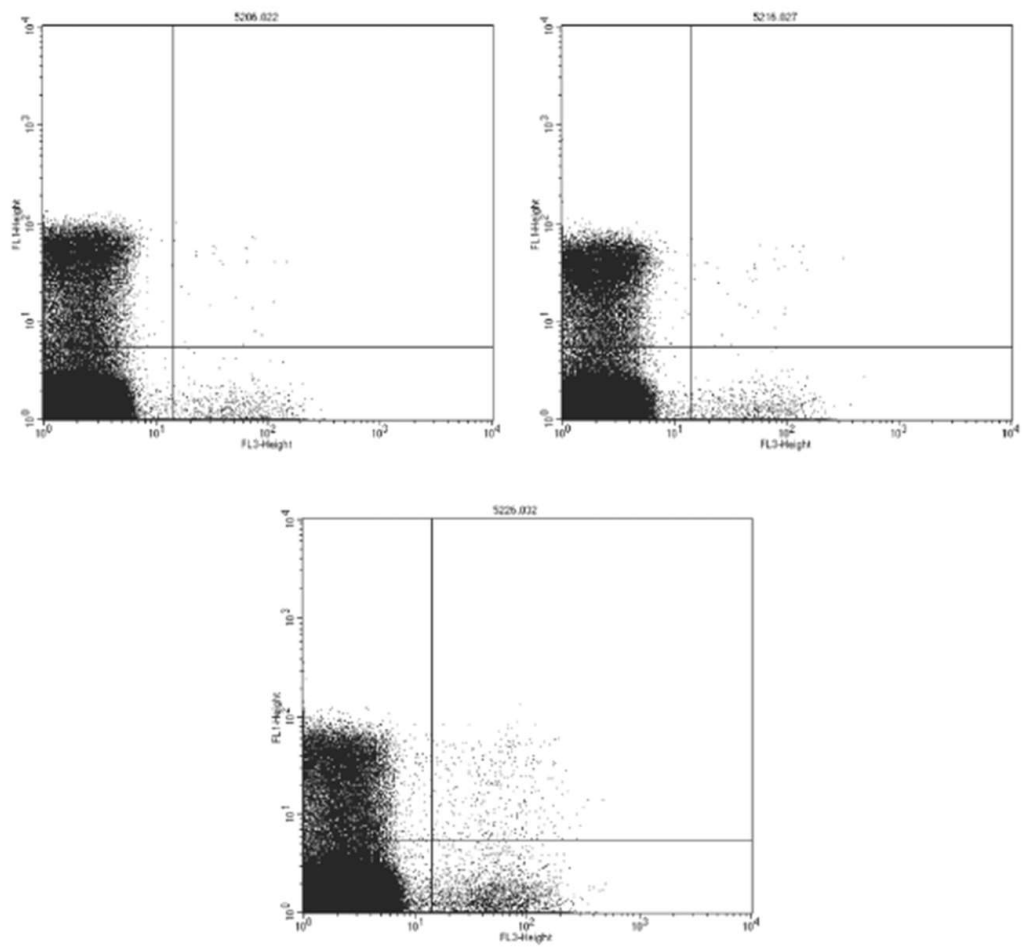

Figure 2B. Three Representative Bivariates (Female). These representative bivariates of test facility submitted Samples 5206, 5216, and 5226 illustrate the resolution of the various erythrocyte populations in mouse peripheral blood: Lower Left quadrant $=$ NCE [cells which are low in green and red fluorescence]; Lower Right $=$ MN-NCE [cells high in red (PI) fluorescence]; Upper Left = CD71 positive RET [cells with green (CD71) fluorescence]; Upper Right = CD71 positive MN-RET [cells with both red and green fluorescence; the population of primary interest for this analysis].

All animals survived administration of the negative control, test substance, or positive control. The initial body weights of all animals were within $+20 \%$ of the mean for each sex. No clinical observations were noted in any animal during the study.

Under the conditions of this study, silk fibroin at the maximum dose of $1000 \mathrm{mg} / \mathrm{kg}$ bodyweight/day did not induce micronucleus formation in the immature erythrocytes of the mouse. As such, silk fibroin is not considered to be genotoxic with respect to micronucleus induction in the in vivo Mouse Erythrocyte Micronucleus Test (Flow Cytometry).

\subsection{Fourteen-Day Repeat Dose Oral Gavage Range-Finding Study}

A 14-day toxicity study was performed in rats to evaluate the clinical effects and establish a maximum tolerated dose of repeated silk fibroin dosing. The test substance was administered in doses of 0,125 , 250, and $500 \mathrm{mg} / \mathrm{kg}$-bw/day (Groups 1-4, respectively). All animals survived test substance administration. There were no clinical observations considered related to test substance administration. Incidental clinical observations included: superficial eschar on the face of one of five Group 2 males and a lesion on the tail of one of five Group 4 males. Neither of these findings was deemed to be test substance related. 
No macroscopic findings were noted in necropsy, which included examination of the external surface of the body, all orifices, musculoskeletal system, and the cranial, thoracic, abdominal, and pelvic cavities with their associated organs and tissues.

Overall and mean weekly body weights (Table 4A) and mean daily body weight gain (Table 4B) for treated male and female rats in Groups 2-4 were comparable to their respective control Group 1 values throughout the study. Mean daily food consumption (Table 4C) and mean food efficiency (Table D) for treated male and female rats in Groups 2-4 were comparable to control Group 1 values on Days 8-15. Under the conditions of the study and based on the toxicological endpoints evaluated, male and female Sprague Dawley rats are expected to tolerate dose levels of $500 \mathrm{mg} / \mathrm{kg}$ bodyweight/day of silk fibroin in a study of longer duration.

Table 4A. Mean body weight \pm SD, g (14-day study)

\begin{tabular}{lccccccccc}
\hline & SF dose & \multicolumn{2}{c}{$0 \mathrm{mg} / \mathrm{kg}$ bw/day } & \multicolumn{2}{c}{$125 \mathrm{mg} / \mathrm{kg}$ bw/day } & \multicolumn{2}{c}{$250 \mathrm{mg} / \mathrm{kg}$ bw/day } & \multicolumn{2}{c}{$500 \mathrm{mg} / \mathrm{kg}$ bw/day } \\
\hline & Day & $\mathrm{M}$ & $\mathrm{F}$ & $\mathrm{M}$ & $\mathrm{F}$ & $\mathrm{M}$ & $\mathrm{F}$ & $\mathrm{M}$ & $\mathrm{F}$ \\
\hline 1 & $236.2 \pm 16.7$ & $151.2 \pm 5.5$ & $237.0 \pm 18.9$ & $151.4 \pm 15.0$ & $233.4 \pm 18.4$ & $152.6 \pm 8.0$ & $236.6 \pm 15.4$ & $154.2 \pm 7.0$ \\
8 & $289.2 \pm 18.3$ & $176.8 \pm 8.6$ & $281.0 \pm 25.3$ & $169.4 \pm 12.7$ & $311.4 \pm 40.6$ & $177.2 \pm 11.3$ & $295.4 \pm 16.1$ & $181.0 \pm 7.4$ \\
15 & $344.8 \pm 30.6$ & $200.6 \pm 11.6$ & $331.8 \pm 36.7$ & $196.2 \pm 9.7$ & $344.0 \pm 29.6$ & $198.4 \pm 13.2$ & $351.6 \pm 21.1$ & $204.8 \pm 9.9$ \\
\hline
\end{tabular}

$\mathrm{n}=20$; $\mathrm{SD}$, standard deviation; $\mathrm{SF}$, silk fibroin; $\mathrm{M}$, male; $\mathrm{F}$, female

Table 4B. Mean body weight gain \pm SD, g/day (14-day study)

\begin{tabular}{lcccccccc}
\hline \multicolumn{1}{c}{$\mathrm{SF}$ dose } & \multicolumn{2}{c}{$0 \mathrm{mg} / \mathrm{kg}$ bw/day } & \multicolumn{2}{c}{$125 \mathrm{mg} / \mathrm{kg}$ bw/day } & \multicolumn{2}{c}{$250 \mathrm{mg} / \mathrm{kg}$ bw/day } & \multicolumn{2}{c}{$500 \mathrm{mg} / \mathrm{kg} \mathrm{bw} / \mathrm{day}$} \\
\hline \multicolumn{1}{c}{ Day } & $\mathrm{M}$ & $\mathrm{F}$ & $\mathrm{M}$ & $\mathrm{F}$ & $\mathrm{M}$ & $\mathrm{F}$ & $\mathrm{M}$ & $\mathrm{F}$ \\
\hline $1 \rightarrow 8$ & $7.57 \pm 2.27$ & $3.66 \pm 0.59$ & $6.29 \pm 1.33$ & $2.57 \pm 1.10$ & $11.14 \pm 6.35$ & $3.51 \pm 0.58$ & $8.40 \pm 0.40$ & $3.83 \pm 0.23$ \\
$8 \rightarrow 15$ & $7.94 \pm 2.20$ & $3.40 \pm 0.55$ & $7.26 \pm 2.48$ & $3.83 \pm 0.80$ & $4.66 \pm 7.42$ & $3.03 \pm 0.36$ & $8.03 \pm 0.88$ & $3.40 \pm 0.57$ \\
\hline
\end{tabular}

$\mathrm{n}=20$; $\mathrm{SD}$, standard deviation; $\mathrm{SF}$, silk fibroin; $\mathrm{M}$, male; $\mathrm{F}$, female

Table 4C. Mean daily food consumption \pm SD, g/day (14-day study)

\begin{tabular}{cccccccccc}
\hline SF dose & \multicolumn{2}{c}{$0 \mathrm{mg} / \mathrm{kg}$ bw/day } & \multicolumn{2}{c}{$125 \mathrm{mg} / \mathrm{kg}$ bw/day } & \multicolumn{2}{c}{$250 \mathrm{mg} / \mathrm{kg}$ bw/day } & \multicolumn{3}{c}{$500 \mathrm{mg} / \mathrm{kg}$ bw/day } \\
\hline Day & M & F & M & F & M & F & M & F \\
\hline $1 \rightarrow 8$ & $35.03 \pm 10.99$ & $38.54 \pm 7.39$ & $34.63 \pm 12.99$ & $38.43 \pm 7.17$ & $34.17 \pm 14.45$ & $37.40 \pm 6.74$ & $37.97 \pm 12.42$ & $26.60^{\mathrm{a}} \pm 9.22$ \\
$8 \rightarrow 15$ & $35.77 \pm 11.62$ & $19.17 \pm 0.89$ & $26.80 \pm 0.40$ & $17.71 \pm 0.72$ & $24.94 \pm 5.40$ & $19.11 \pm 0.81$ & $29.20 \pm 1.49$ & $23.03^{\mathrm{a}} \pm 3.81$ \\
\hline
\end{tabular}

$\mathrm{n}=20$; SD, standard deviation; $\mathrm{SF}$, silk fibroin; $\mathrm{M}$, male; $\mathrm{F}$, female ${ }^{\mathrm{a}} \mathrm{p}<0.05$

Table 4D. Mean food efficiency \pm SD, $\mathrm{mg} / \mathrm{kg}$ bw/day (14-day study)

\begin{tabular}{ccccccccc}
\hline \multicolumn{1}{c}{ SF dose } & \multicolumn{2}{c}{$0 \mathrm{mg} / \mathrm{kg} \mathrm{bw} /$ day } & \multicolumn{2}{c}{$125 \mathrm{mg} / \mathrm{kg}$ bw/day } & \multicolumn{2}{c}{$250 \mathrm{mg} / \mathrm{kg} \mathrm{bw} / \mathrm{day}$} & \multicolumn{2}{c}{$500 \mathrm{mg} / \mathrm{kg} \mathrm{bw} / \mathrm{day}$} \\
\hline \multirow{2}{*}{ Day } & $\mathrm{M}$ & $\mathrm{F}$ & $\mathrm{M}$ & $\mathrm{F}$ & $\mathrm{M}$ & $\mathrm{F}$ & $\mathrm{M}$ & $\mathrm{F}$ \\
\multirow{2}{*}{$1 \rightarrow 8$} & $0.243 \pm$ & $0.098 \pm$ & $0.193 \pm$ & $0.066^{\mathrm{a}} \pm$ & $0.393 \pm$ & $0.095^{\mathrm{a}} \pm$ & $0.242 \pm$ & $0.159^{\mathrm{a}} \pm$ \\
& 0.118 & 0.025 & 0.048 & 0.027 & 0.320 & 0.019 & 0.078 & 0.056 \\
\multirow{2}{*}{$8 \rightarrow 15$} & $0.229 \pm$ & $0.178 \pm$ & $0.271 \pm$ & $0.216^{\mathrm{a}} \pm$ & $0.167 \pm$ & $0.159^{\mathrm{a}} \pm$ & $0.276 \pm$ & $0.148^{\mathrm{a}} \pm$ \\
& 0.059 & 0.030 & 0.093 & 0.046 & 0.320 & 0.022 & 0.034 & 0.018 \\
\hline
\end{tabular}

$\mathrm{n}=20$; SD, standard deviation; SF, silk fibroin; $\mathrm{M}$, male; $\mathrm{F}$, female ${ }^{\mathrm{a}} \mathrm{p}<0.05$ 
16 of 30

\subsection{Twenty-Eight-Day Repeat-Dose Oral Toxicity Study}

Following the 14-day study, an expanded 28-day study was performed to evaluate toxicity of repeated silk fibroin consumption. Due to the lack of adverse clinical effects at all dose ranges in the 14-day study, a maximum tolerated dose could not be established. The same dose range was therefore used in the 28-day study: 0, 125, 250, and $500 \mathrm{mg} / \mathrm{kg}$ bodyweight/day (Groups 1-4, respectively).

All animals survived test substance administration and there were no unscheduled deaths among the animals submitted for histopathological evaluation. The gross findings at terminal sacrifice were considered incidental, of the nature commonly observed in rats, and/or were of similar incidence in control and dosed rats. Incidental observations included: macroscopic unilateral (right) small and/or flaccid testes correlated microscopically with testicular atrophy in one Group 1 and one Group 2 males; macroscopic findings of small right epididymis that microscopically correlated with epididymal atrophy in one Group 1 male. The macroscopic findings were not considered related to administration of silk fibroin.

No silk fibroin-related microscopic findings were noted in terminal sacrifice animals. The microscopic findings observed were considered incidental (background findings), of the nature commonly observed in rats, and/or were of similar incidence and severity in the control and dosed animals and were not considered related to the administration of silk fibroin.

Overall and mean weekly body weights (Table 5A) and mean daily body weight gain (5B) for treated male and female rats in Groups 2-4 were comparable to their respective control Group 1 values throughout the study. Mean daily food consumption (Table 6A) and mean food efficiency (Table 6B) for treated male and female rats in Groups 2-4 were comparable to control Group 1 values throughout the study. Hematology and coagulation (Table 7A), serum chemistry (Table 7B), and urinalysis results (Table 7C) values fell within expected ranges and raise no concern regarding the effects of ingested silk fibroin.

Table 5A. Mean body weight \pm SD, g (28-day study)

\begin{tabular}{lccccccccc}
\hline \multicolumn{2}{c}{ SF dose } & \multicolumn{2}{c}{$0 \mathrm{mg} / \mathrm{kg}$ bw/day } & \multicolumn{2}{c}{$125 \mathrm{mg} / \mathrm{kg}$ bw/day } & \multicolumn{2}{c}{$250 \mathrm{mg} / \mathrm{kg} \mathrm{bw} / \mathrm{day}$} & \multicolumn{2}{c}{$500 \mathrm{mg} / \mathrm{kg} \mathrm{bw} / \mathrm{day}$} \\
\hline \multicolumn{2}{c}{ Day } & $\mathrm{M}$ & $\mathrm{F}$ & $\mathrm{M}$ & $\mathrm{F}$ & $\mathrm{M}$ & $\mathrm{F}$ & $\mathrm{M}$ & $\mathrm{F}$ \\
\hline 1 & $231.9 \pm 12.5$ & $204.2 \pm 13.9$ & $232.2 \pm 12.3$ & $204.9 \pm 12.8$ & $233.0 \pm 13.6$ & $205.3 \pm 13.3$ & $231.7 \pm 12.6$ & $205.9 \pm 11.8$ \\
8 & $276.6 \pm 17.3$ & $224.3 \pm 14.2$ & $282.2 \pm 11.6$ & $222.6 \pm 16.7$ & $284.5 \pm 16.0$ & $226.4 \pm 16.0$ & $289.9 \pm 16.9$ & $221.4 \pm 14.8$ \\
15 & $332.1 \pm 17.4$ & $243.2 \pm 16.0$ & $327.5 \pm 14.2$ & $240.9 \pm 23.7$ & $335.2 \pm 14.4$ & $241.5 \pm 18.0$ & $340.8 \pm 20.1$ & $239.7 \pm 16.2$ \\
22 & $380.2 \pm 26.5$ & $256.3 \pm 17.2$ & $372.8 \pm 15.6$ & $252.4 \pm 26.1$ & $379.1 \pm 18.9$ & $257.0 \pm 19.9$ & $392.6 \pm 20.5$ & $255.7 \pm 21.5$ \\
29 & $428.6 \pm 26.9$ & $268.6 \pm 18.4$ & $410.1 \pm 18.7$ & $267.0 \pm 30.1$ & $416.6 \pm 18.4$ & $269.3 \pm 23.9$ & $438.2 \pm 22.3$ & $273.2 \pm 25.3$ \\
\hline
\end{tabular}

$\mathrm{n}=20$; SD, standard deviation; $\mathrm{SF}$, silk fibroin; $\mathrm{M}$, male; $\mathrm{F}$, female

Table 5B. Mean body weight gain \pm SD, g/day (28-day study)

\begin{tabular}{lcccccccc}
\hline \multicolumn{1}{c}{ SF dose } & \multicolumn{2}{c}{$0 \mathrm{mg} / \mathrm{kg}$ bw/day } & \multicolumn{2}{c}{$125 \mathrm{mg} / \mathrm{kg} \mathrm{bw} /$ day } & \multicolumn{2}{c}{$250 \mathrm{mg} / \mathrm{kg} \mathrm{bw} /$ day } & \multicolumn{2}{c}{$500 \mathrm{mg} / \mathrm{kg} \mathrm{bw} / \mathrm{day}$} \\
\hline \multicolumn{1}{c}{ Day } & $\mathrm{M}$ & $\mathrm{F}$ & $\mathrm{M}$ & $\mathrm{F}$ & $\mathrm{M}$ & $\mathrm{F}$ & $\mathrm{M}$ & $\mathrm{F}$ \\
\hline $1 \rightarrow 8$ & $6.39 \pm 1.52$ & $2.87 \pm 0.82$ & $7.14 \pm 1.23$ & $2.53 \pm 0.96$ & $7.36 \pm 0.84$ & $3.01 \pm 0.68$ & $8.31^{\mathrm{b}} \pm 1.17$ & $2.21 \pm 1.07$ \\
$8 \rightarrow 15$ & $7.93 \pm 2.14$ & $2.70 \pm 1.42$ & $6.47 \pm 0.62$ & $2.61 \pm 1.38$ & $7.24 \pm 0.92$ & $2.16 \pm 1.11$ & $7.27 \pm 1.03$ & $2.61 \pm 1.07$ \\
$15 \rightarrow 22$ & $6.87 \pm 1.93$ & $1.87 \pm 1.37$ & $6.47 \pm 1.49$ & $1.64 \pm 1.25$ & $6.27 \pm 0.93$ & $2.21 \pm 0.91$ & $7.40 \pm 0.74$ & $2.29 \pm 1.25$ \\
$22 \rightarrow 29$ & $6.91 \pm 1.55$ & $1.76 \pm 1.35$ & $5.33^{\mathrm{a}} \pm 0.81$ & $2.09 \pm 1.03$ & $5.36^{\mathrm{a}} \pm 0.93$ & $1.76 \pm 1.16$ & $6.51 \pm 1.42$ & $2.50 \pm 1.65$ \\
\hline
\end{tabular}

$\mathrm{n}=20$; SD, standard deviation; SF, silk fibroin; M, male; F, female; ${ }^{a} p<0.05$; ${ }^{b} p<0.01$ 
17 of 30

Table 6A. Mean daily food consumption \pm SD, g/day (28-day study)

\begin{tabular}{ccccccccc}
\hline \multicolumn{1}{c}{ SF dose } & \multicolumn{2}{c}{$0 \mathrm{mg} / \mathrm{kg} \mathrm{bw} / \mathrm{day}$} & \multicolumn{2}{c}{$125 \mathrm{mg} / \mathrm{kg}$ bw/day } & \multicolumn{2}{c}{$250 \mathrm{mg} / \mathrm{kg}$ bw/day } & \multicolumn{2}{c}{$500 \mathrm{mg} / \mathrm{kg} \mathrm{bw} / \mathrm{day}$} \\
\hline \multicolumn{1}{c}{ Day } & $\mathrm{M}$ & $\mathrm{F}$ & $\mathrm{M}$ & $\mathrm{F}$ & $\mathrm{M}$ & $\mathrm{F}$ & $\mathrm{M}$ & $\mathrm{F}$ \\
\hline $1 \rightarrow 8$ & $24.69^{\mathrm{b}} \pm 1.60$ & $20.23 \pm 1.23$ & $25.00 \pm 1.09$ & $19.71 \pm 0.86$ & $25.89 \pm 1.71$ & $19.77 \pm 1.46$ & $26.67 \pm 1.27$ & $20.90 \pm 1.60$ \\
$8 \rightarrow 15$ & $28.06 \pm 1.83$ & $21.26 \pm 1.39$ & $27.34 \pm 0.71$ & $21.01 \pm 0.77$ & $28.93 \pm 1.34$ & $20.43 \pm 1.53$ & $28.77 \pm 1.46$ & $21.43 \pm 0.71$ \\
$15 \rightarrow 22$ & $28.49 \pm 1.87$ & $19.87 \pm 1.26$ & $27.61 \pm 2.08$ & $19.44 \pm 1.66$ & $28.29 \pm 1.12$ & $19.71 \pm 1.29$ & $29.54 \pm 0.96$ & $20.96 \pm 1.75$ \\
$22 \rightarrow 29$ & $29.34 \pm 2.35$ & $20.04 \pm 1.58$ & $27.33^{\mathrm{a}} \pm 1.14$ & $19.89 \pm 1.40$ & $28.49 \pm 1.05$ & $20.06 \pm 1.51$ & $30.46 \pm 1.29$ & $21.94 \pm \pm 1.53$ \\
\hline
\end{tabular}

$\mathrm{n}=20$; SD, standard deviation; $\mathrm{SF}$, silk fibroin; $\mathrm{M}$, male; $\mathrm{F}$, female

a $p<0.05$

${ }^{\mathrm{b}} p<0.01$

Table 6B. Mean food efficiency \pm SD, mg/kg/day (28-day study)

\begin{tabular}{|c|c|c|c|c|c|c|c|c|}
\hline \multirow{2}{*}{$\frac{\text { SF dose }}{\text { Day }}$} & \multicolumn{2}{|c|}{$0 \mathrm{mg} / \mathrm{kg}$ bw/day } & \multicolumn{2}{|c|}{$125 \mathrm{mg} / \mathrm{kg}$ bw/day } & \multicolumn{2}{|c|}{$250 \mathrm{mg} / \mathrm{kg}$ bw/day } & \multicolumn{2}{|c|}{500 mg/kg bw/day } \\
\hline & M & $\mathrm{F}$ & M & $\mathrm{F}$ & M & $\mathrm{F}$ & M & $\mathrm{F}$ \\
\hline \multirow{2}{*}{$1 \rightarrow 8$} & $0.258 \pm$ & $0.142 \pm$ & $0.286 \pm$ & $0.131 \pm$ & $0.284 \pm$ & $0.152 \pm$ & $0.311 \pm$ & $0.106 \pm$ \\
\hline & 0.055 & 0.041 & 0.048 & 0.052 & 0.028 & 0.033 & 0.038 & 0.050 \\
\hline \multirow{2}{*}{$8 \rightarrow 15$} & $0.281 \pm$ & $0.128 \pm$ & $0.237 \pm$ & $0.125 \pm$ & $0.250 \pm$ & $0.104 \pm$ & $0.252 \pm$ & $0.122 \pm$ \\
\hline & 0.062 & 0.068 & 0.025 & 0.067 & 0.029 & 0.050 & 0.030 & 0.047 \\
\hline \multirow{2}{*}{$15 \rightarrow 22$} & $0.239 \pm$ & $0.092 \pm$ & $0.235 \pm$ & $0.081 \pm$ & $0.222 \pm$ & $0.113 \pm$ & $0.250 \pm$ & $0.109 \pm$ \\
\hline & 0.060 & 0.065 & 0.058 & 0.061 & 0.035 & 0.047 & 0.022 & 0.061 \\
\hline \multirow{2}{*}{$22 \rightarrow 29$} & $0.236 \pm$ & $0.087 \pm$ & $0.196 \pm$ & $0.106 \pm$ & $0.189 \pm$ & $0.085 \pm$ & $0.213 \pm$ & $0.111 \pm$ \\
\hline & 0.054 & 0.067 & 0.036 & 0.053 & 0.036 & 0.052 & 0.042 & 0.067 \\
\hline
\end{tabular}

$\mathrm{n}=20$; SD, standard deviation; SF, silk fibroin; $\mathrm{M}$, male; $\mathrm{F}$, female

Table 7A. Hematology and coagulation, mean \pm SD (28-day study)

\begin{tabular}{|c|c|c|c|c|c|c|c|c|}
\hline SF dose & \multicolumn{2}{|c|}{$0 \mathrm{mg} / \mathrm{kg}$ bw/day } & \multicolumn{2}{|c|}{$125 \mathrm{mg} / \mathrm{kg}$ bw/day, } & \multicolumn{2}{|c|}{$250 \mathrm{mg} / \mathrm{kg}$ bw/day } & \multicolumn{2}{|c|}{500 mg/kg bw/day } \\
\hline Parameter & M & $\mathrm{F}$ & M & $\mathrm{F}$ & M & $\mathrm{F}$ & M & $\mathrm{F}$ \\
\hline \multirow{2}{*}{$\mathrm{RBC}, \times 10^{6} / \mu \mathrm{L}$} & $8.287 \pm$ & $8.349 \pm$ & $8.341 \pm$ & $8.363 \pm$ & $8.245 \pm$ & $8.216 \pm$ & $7.909 \pm$ & $8.265 \pm$ \\
\hline & 0.3027 & 0.4709 & 0.6971 & 0.6045 & 0.5894 & 0.6070 & 0.4059 & 0.3784 \\
\hline \multirow{2}{*}{ HGB, g/dL } & $15.73 \pm$ & $15.46 \pm$ & $15.67 \pm$ & $15.35 \pm$ & $15.93 \pm$ & $15.23 \pm$ & $15.38 \pm$ & $15.32 \pm$ \\
\hline & 0.403 & 0.789 & 0.998 & 0.902 & 0.641 & 1.215 & 0.553 & 0.646 \\
\hline \multirow{2}{*}{$\mathrm{HCT}, \%$} & $52.35 \pm$ & $50.81 \pm$ & $52.21 \pm$ & $50.19 \pm$ & $52.72 \pm$ & $49.67 \pm$ & $51.02 \pm$ & $50.92 \pm$ \\
\hline & 1.855 & 2.824 & 4.273 & 3.273 & 2.391 & 4.283 & 2.825 & 1.712 \\
\hline \multirow{2}{*}{$\mathrm{MCV}, \mathrm{fL}$} & $63.39 \pm$ & $60.86 \pm$ & $62.61 \pm$ & $60.08 \pm$ & $64.22 \pm$ & $60.44 \pm$ & $64.56 \pm$ & $61.65 \pm$ \\
\hline & 2.197 & 0.947 & 1.149 & 1.483 & 1.825 & 1.308 & 1.366 & 2.022 \\
\hline \multirow{2}{*}{$\mathrm{MCH}, \mathrm{pg}$} & $19.00 \pm$ & $18.51 \pm$ & $18.83 \pm$ & $18.35 \pm$ & $19.35 \pm$ & $18.53 \pm$ & $19.45 \pm$ & $18.55 \pm$ \\
\hline & 0.680 & 0.328 & 0.680 & 0.570 & 0.747 & 0.442 & 0.570 & 0.595 \\
\hline \multirow{2}{*}{ RDW, \% } & $12.21 \pm$ & $11.30 \pm$ & $12.10 \pm$ & $11.01 \pm$ & $11.99 \pm$ & $11.35 \pm$ & $12.48 \pm$ & $11.20 \pm$ \\
\hline & 0.396 & 0.422 & 0.356 & 0.351 & 0.532 & 0.430 & 0.567 & 0.467 \\
\hline \multirow{2}{*}{ ARET, $x 10^{3} / \mu \mathrm{L}$} & $226.6 \pm$ & $180.7 \pm$ & $206.4 \pm$ & $167.9 \pm$ & $224.4 \pm$ & $178.4 \pm$ & $243.2 \pm$ & $176.2 \pm$ \\
\hline & 36.52 & 44.86 & 29.50 & 25.32 & 28.11 & 38.27 & 52.97 & 36.59 \\
\hline
\end{tabular}


18 of 30

$\begin{array}{lcccccccc} & 1182.1 \pm & 1092.7 \pm & 1192.6 \pm & 1084.3 \pm & 984.3 \pm & 1015.4 \pm & 1142.8 \pm & 975.7 \pm \\ \text { PLT, } x 103 / \mu \mathrm{L} & 114.77 & 133.06 & 90.93 & 82.26 & 335.49 & 160.48 & 110.00 & 207.49 \\ \text { WBC, } \times 103 / \mu \mathrm{L} & 9.836 \pm & 7.684 \pm & 9.670 \pm & 7.788 \pm & 8.643 \pm & 6.465 \pm & 8.978 \pm & 7.404 \pm \\ & 2.2150 & 2.3797 & 2.9527 & 1.6943 & 1.6761 & 0.9602 & 1.9173 & 2.1553 \\ \text { MCHC, g/dL } & 30.01 \pm & 30.14 \pm & 30.00 \pm & 30.52 \pm & 30.18 \pm & 30.61 \pm & 27.42 \pm & 30.09 \pm \\ & 0.540 & 0.583 & 0.751 & 0.569 & 0.437 & 0.507 & 8.459 & 0.458 \\ \text { APTT, sec } & 16.5 \pm & 16.1 \pm & 16.8 \pm & 16.2 \pm & 18.7 \pm & 15.9 \pm & 18.5 \pm & 16.4 \pm \\ & 1.24 & 1.27 & 3.09 & 1.12 & 3.03 & 1.19 & 3.77 & 1.46 \\ \text { PT, sec } & 9.6 \pm & 9.1 \pm & 9.5 \pm & 9.0 \pm & 9.4 \pm & 9.1 \pm & 9.6 \pm & 9.0 \pm\end{array}$

$\mathrm{n}=40$; $\mathrm{SD}$, standard deviation; $\mathrm{SF}$, silk fibroin; $\mathrm{M}$, male; $\mathrm{F}$, female; $\mathrm{RBC}$, erythrocyte count; $\mathrm{HGB}$, hemoglobin concentration; $\mathrm{HCT}$, hematocrit; $\mathrm{MCV}$, mean corpuscular volume; $\mathrm{MCH}$, mean corpuscular hemoglobin; RDW, red cell distribution width; ARET, absolute reticulocyte count; PLT, platelet count; WBC, total white blood cell; MCHC, mean corpuscular hemoglobin concentration; APTT, activated partial thromboplastin time; PT, prothrombin time

Table 7B. Serum chemistry, mean \pm SD (28-day study)

\begin{tabular}{|c|c|c|c|c|c|c|c|c|}
\hline \multirow{2}{*}{$\begin{array}{l}\text { SF dose } \\
\text { Parameter }\end{array}$} & \multicolumn{2}{|c|}{$0 \mathrm{mg} / \mathrm{kg} \mathrm{bw} /$ day } & \multicolumn{2}{|c|}{$125 \mathrm{mg} / \mathrm{kg}$ bw/day } & \multicolumn{2}{|c|}{$250 \mathrm{mg} / \mathrm{kg}$ bw/day } & \multicolumn{2}{|c|}{$500 \mathrm{mg} / \mathrm{kg}$ bw/day } \\
\hline & M & $\mathrm{F}$ & M & $\mathrm{F}$ & $\mathrm{M}$ & $\mathrm{F}$ & $\mathrm{M}$ & $\mathrm{F}$ \\
\hline \multirow{2}{*}{ AST (U/L) } & $80.0 \pm$ & $82.9 \pm$ & $75.5 \pm$ & $80.7 \pm$ & $65.7 \pm$ & $102.2 \pm$ & $68.1 \pm$ & $94.9 \pm$ \\
\hline & 14.89 & 29.00 & 14.95 & 22.62 & 13.17 & 67.16 & 9.40 & 28.86 \\
\hline \multirow{2}{*}{$\operatorname{ALT}(\mathrm{U} / \mathrm{L})$} & $27.100 \pm$ & $27.700 \pm$ & $28.500 \pm$ & $23.200 \pm$ & $24.000 \pm$ & $37.300 \pm$ & $24.900 \pm$ & $44.100 \pm$ \\
\hline & 6.2796 & 7.1964 & 6.0964 & 4.2111 & 5.2493 & 28.4412 & 4.2282 & 39.1619 \\
\hline \multirow{2}{*}{$\mathrm{SDH}(\mathrm{U} / \mathrm{L})$} & $9.28 \pm$ & $7.36 \pm$ & $7.85 \pm$ & $5.65 \pm$ & $8.83 \pm$ & $18.16 \pm$ & $9.96 \pm$ & $13.69 \pm$ \\
\hline & 2.033 & 2.878 & 2.618 & 2.170 & 2.503 & 30.935 & 3.302 & 12.405 \\
\hline \multirow{2}{*}{$\operatorname{ALKP}(\mathrm{U} / \mathrm{L})$} & $132.3 \pm$ & $62.6 \pm$ & $151.1 \pm$ & $64.3 \pm$ & $133.5 \pm$ & $65.1 \pm$ & $112.7 \pm$ & $70.8 \pm$ \\
\hline & 27.24 & 20.72 & 23.82 & 16.59 & 25.71 & 16.91 & 24.14 & 20.66 \\
\hline \multirow{2}{*}{ TBIL (mg/dL) } & $0.066 \pm$ & $0.077 \pm$ & $0.062 \pm$ & $0.085 \pm$ & $0.064 \pm$ & $0.082 \pm$ & $0.070 \pm$ & $0.090 \pm$ \\
\hline & 0.0097 & 0.0106 & 0.0155 & 0.0190 & 0.0126 & 0.0175 & 0.0226 & 0.0330 \\
\hline \multirow{2}{*}{ BUN (mg/dL) } & $12.8 \pm$ & $13.9 \pm$ & $13.3 \pm$ & $15.1 \pm$ & $12.6 \pm$ & $14.0 \pm$ & $12.8 \pm$ & $14.3 \pm$ \\
\hline & 1.48 & 2.02 & 1.34 & 2.08 & 1.90 & 2.11 & 0.92 & 1.34 \\
\hline \multirow{2}{*}{ CREA (mg/dL) } & $0.163 \pm$ & $0.201 \pm$ & $0.170 \pm$ & $0.230 \pm$ & $0.174 \pm$ & $0.199 \pm$ & $0.170 \pm$ & $0.225 \pm$ \\
\hline & 0.0231 & 0.0357 & 0.0452 & 0.0488 & 0.0443 & 0.0387 & 0.0346 & 0.0433 \\
\hline \multirow{2}{*}{ CHOL $(\mathrm{mg} / \mathrm{dL})$} & $56.9 \pm$ & $78.2 \pm$ & $60.9 \pm$ & $75.1 \pm$ & $63.1 \pm$ & $72.3 \pm$ & $56.0 \pm$ & $75.0 \pm$ \\
\hline & 14.69 & 15.82 & 10.21 & 19.81 & 10.65 & 12.23 & 14.35 & 19.76 \\
\hline \multirow{2}{*}{ TRIG (mg/dL) } & $45.5 \pm$ & $45.4 \pm$ & $40.7 \pm$ & $37.9 \pm$ & $43.6 \pm$ & $42.2 \pm$ & $52.4 \pm$ & $41.3 \pm$ \\
\hline & 9.99 & 16.63 & 9.73 & 12.99 & 12.02 & 9.82 & 19.07 & 17.70 \\
\hline \multirow{2}{*}{ GLUC (mg/dL) } & $135.2 \pm$ & $140.6 \pm$ & $113.4 \pm$ & $121.8 \pm$ & $119.3 \pm$ & $131.6 \pm$ & $123.4 \pm$ & $121.8 \pm$ \\
\hline & 36.30 & 36.79 & 25.50 & 41.74 & 31.11 & 31.39 & 22.19 & 35.93 \\
\hline \multirow{2}{*}{$\mathrm{TP}(\mathrm{g} / \mathrm{dL})$} & $5.72 \pm$ & $6.93 \pm$ & $5.83 \pm$ & $6.74 \pm$ & $5.88 \pm$ & $6.49 \pm$ & $5.77 \pm$ & $6.84 \pm$ \\
\hline & 0.405 & 0.593 & 0.327 & 0.595 & 0.301 & 0.821 & 0.564 & 0.585 \\
\hline \multirow{2}{*}{$\operatorname{ALB}(\mathrm{g} / \mathrm{dL})$} & $3.64 \pm$ & $4.86 \pm$ & $3.76 \pm$ & $4.68 \pm$ & $3.78 \pm$ & $4.50 \pm$ & $3.76 \pm$ & $4.69 \pm$ \\
\hline & 0.347 & 0.548 & 0.227 & 0.527 & 0.322 & 0.655 & 0.344 & 0.398 \\
\hline
\end{tabular}




\begin{tabular}{lccccccccc} 
& $2.08 \pm$ & $2.07 \pm$ & $2.07 \pm$ & $2.06 \pm$ & $2.10 \pm$ & $1.99 \pm$ & $2.01 \pm$ & $2.15 \pm$ \\
GLOB (g/dL) & 0.235 & 0.125 & 0.125 & 0.237 & 0.082 & 0.360 & 0.251 & 0.276 \\
CA (mg/dL) & $10.54 \pm$ & $10.46 \pm$ & $10.46 \pm$ & $10.30 \pm$ & $10.22 \pm$ & $10.07 \pm$ & $10.16 \pm$ & $10.51 \pm$ \\
& 0.826 & 1.256 & 0.648 & 0.874 & 0.877 & 0.815 & 0.785 & 1.064 \\
PHOS (mg/dL) & $9.47 \pm$ & $7.88 \pm$ & $9.61 \pm$ & $8.25 \pm$ & $9.30 \pm$ & $7.88 \pm$ & $9.46 \pm$ & $8.37 \pm$ \\
& 0.732 & 0.666 & 1.119 & 1.033 & 0.847 & 1.214 & 0.738 & 1.024 \\
$\mathrm{Na}(\mathrm{mmol} / \mathrm{L})$ & $140.4 \pm$ & $133.5 \pm$ & $140.9 \pm$ & $134.4 \pm$ & $139.7 \pm$ & $136.4 \pm$ & $140.0 \pm$ & $136.1 \pm$ \\
& 3.41 & 6.00 & 3.00 & 6.00 & 2.75 & 6.20 & 3.46 & 5.26 \\
$\mathrm{~K}(\mathrm{mmol} / \mathrm{L})$ & $6.997 \pm$ & $6.432 \pm$ & $7.426 \pm$ & $7.130 \pm$ & $7.499 \pm$ & $7.561 \pm$ & $7.076 \pm$ & $7.022 \pm$ \\
& 1.0184 & 0.6382 & 1.6202 & 1.9689 & 0.8967 & 2.4808 & 0.9773 & 1.1857 \\
$\mathrm{Cl}(\mathrm{mmol} / \mathrm{L})$ & $98.67 \pm$ & $94.73 \pm$ & $99.18 \pm$ & $95.67 \pm$ & $98.57 \pm$ & $97.98 \pm$ & $98.60 \pm$ & $96.78 \pm$ \\
& 2.320 & 4.513 & 2.523 & 5.265 & 1.754 & 3.721 & 2.720 & 3.192 \\
\hline
\end{tabular}

$\mathrm{n}=40 ; \mathrm{SD}$, standard deviation; $\mathrm{SF}$, silk fibroin; $\mathrm{M}$, male; $\mathrm{F}$, female; $\mathrm{AST}$, serum aspartate aminotransferase; ALT, serum alanine aminotransferase; SDH, sorbitol dehydrogenase; ALKP, alkaline phosphatase; TBIL, total bilirubin; BUN, urea nitrogen; CREA, blood creatinine; $\mathrm{CHOL}$, total cholesterol; TRIG, triglycerides; GLUC, fasting glucose; TP, total serum protein; ALB, albumin; GLOB, globulin; CA, calcium; PHOS, inorganic phosphorus; NA, sodium; $\mathrm{K}$, potassium; $\mathrm{Cl}$, chloride

Table 7C. Urinalysis, mean \pm SD (28-day study)

\begin{tabular}{lccccccccc}
\hline SF dose & \multicolumn{2}{c}{$0 \mathrm{mg} / \mathrm{kg}$ bw/day } & \multicolumn{2}{c}{$125 \mathrm{mg} / \mathrm{kg}$ bw/day } & \multicolumn{2}{c}{$250 \mathrm{mg} / \mathrm{kg}$ bw/day } & \multicolumn{3}{c}{$500 \mathrm{mg} / \mathrm{kg}$ bw/day } \\
\hline Parameter & $\mathrm{M}$ & $\mathrm{F}$ & $\mathrm{M}$ & $\mathrm{F}$ & $\mathrm{M}$ & $\mathrm{F}$ & $\mathrm{M}$ & $\mathrm{F}$ \\
\hline \multirow{2}{*}{$\mathrm{URO}(\mathrm{EU} / \mathrm{dL})$} & $0.20 \pm$ & $0.20 \pm$ & $0.20 \pm$ & $0.20 \pm$ & $0.20 \pm$ & $0.20 \pm$ & $0.20 \pm$ & $0.20 \pm$ \\
& 0.000 & 0.000 & 0.000 & 0.000 & 0.000 & 0.000 & 0.000 & 0.000 \\
$\mathrm{pH}$ & $6.70 \pm$ & $6.44 \pm$ & $6.65 \pm$ & $6.94 \pm$ & $6.75 \pm$ & $6.55 \pm$ & $6.65 \pm$ & $6.39 \pm$ \\
& 0.350 & 0.300 & 0.474 & 0.563 & 0.354 & 0.438 & 0.242 & 0.220 \\
SG & $1.0245 \pm$ & $1.0178 \pm$ & $1.0240 \pm$ & $1.0213 \pm$ & $1.0240 \pm$ & $1.0215 \pm$ & $1.0235 \pm$ & $1.0256 \pm$ \\
& 0.00643 & 0.00667 & 0.00658 & 0.00835 & 0.00568 & 0.00626 & 0.00784 & 0.00682 \\
UVOL (mL) & $8.76 \pm$ & $10.42 \pm$ & $9.16 \pm$ & $6.70 \pm$ & $10.03 \pm$ & $8.39 \pm$ & $10.18 \pm$ & $7.44 \pm$ \\
& 11.148 & 6.654 & 5.734 & 5.489 & 6.790 & 7.279 & 5.115 & 6.146 \\
UPRO (mg/dL) & $32.5 \pm$ & $1.7 \pm$ & $32.0 \pm$ & $23.8 \pm$ & $19.5 \pm$ & $10.5 \pm$ & $19.5 \pm$ & $15.0 \pm$ \\
& 25.74 & 5.00 & 37.87 & 33.25 & 10.12 & 14.23 & 12.35 & 12.99 \\
\hline
\end{tabular}

$\mathrm{n}=40$; SD, standard deviation; SF, silk fibroin; M, male; F, female; URO, urobilinogen; SG, specific; UVOL, volume; UPRO, protein

\subsection{Digestion \& Allergenicity Studies}

\subsubsection{Pepsin Digestion}

Stained SDS-PAGE gels were generated for silk fibroin digestion at a ratio of 10 activity units per $\mu \mathrm{g}$ (Figure 3A), 1 activity unit per $\mu$ g (Figure 3B), and a reference serial dilution (Figure 4). SDS-PAGE gels showing digestion of a positive control protein to confirm pepsin activity (BSA) can be found in Figure S1 of Supplementary Information. Confirmation of stability of pepsin, silk fibroin, and BSA in SGF can be found in Figure S2. A stained gel of this digestion experiment demonstrated that silk 
fibroin was stable in acid alone (Figure S2, Lanes E-F) but was rapidly digested by pepsin at a ratio of 10:1 in under 2 minutes (Figure 3A, Lane B), most closely resembling Figure 4, Lane E (93\% digestion) in the LOD reference gel. At a ratio of 1 activity unit per microgram, a significant quantity of the silk fibroin was digested in less than five minutes, determined by comparing the band intensity of silk fibroin at 5 minutes in pepsin (Figure 3B, Lane C) to the band intensity representing $75 \%$ digested protein (Figure 4, Lane C) in the LOD reference gel. By 60 minutes (Figure 3B, Lane $\mathrm{G})$, the majority of the silk fibroin was digested with a band intensity closely resembling Figure 4, Lane E (93\% digestion). Per CODEX (2003) guidelines, based on the degree of digestion at both pepsin:protein ratios, as well as the lack of degradation bands resulting from digestion, no added concern of allergenicity risk was found [19].

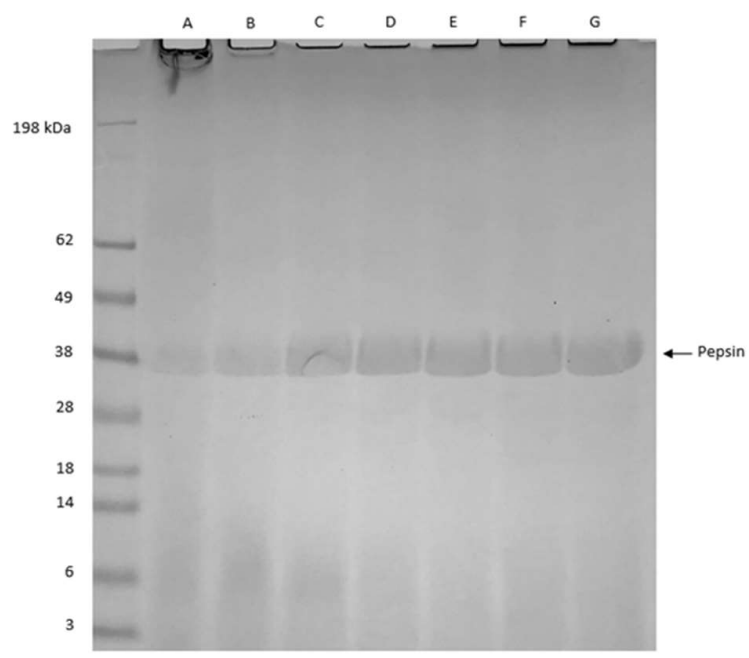

Figure 3A. Pepsin Digestion of Silk Fibroin at a ratio of 10 activity units per microgram test protein. Silk fibroin at $350 \mu \mathrm{g}$ run with pepsin in SGF sampled at various time points. Lanes A-G contain samples of silk fibroin digested with pepsin at 0 (Lane A), 2 (Lane B), 5 (Lane C), 10 (Lane D), 20 (Lane E), 30 (Lane F), and 60 (Lane G) minute intervals.

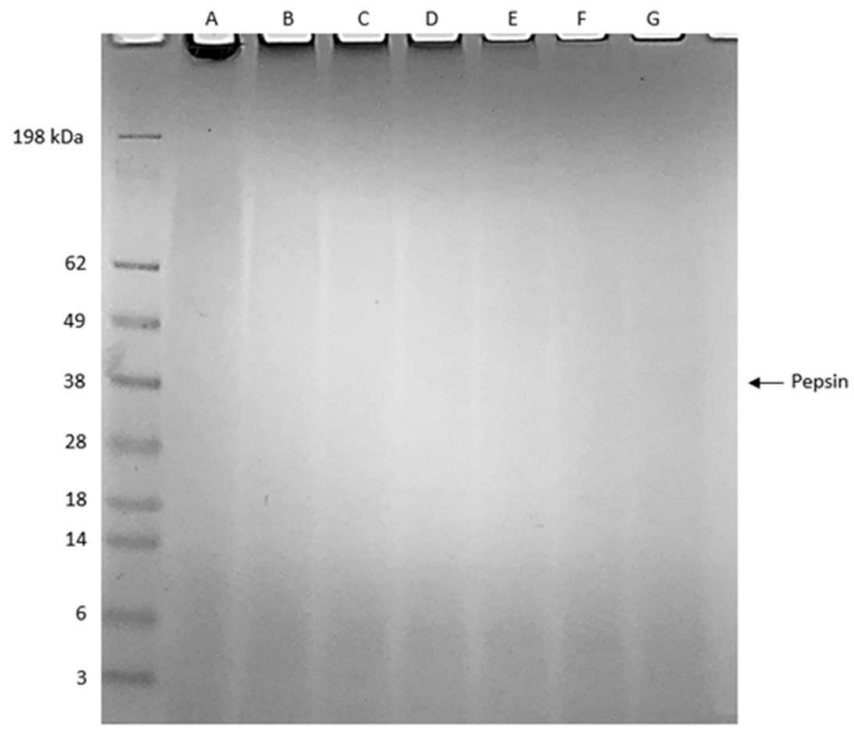

Figure 3B. Pepsin Digestion of Silk Fibroin at a ratio of 1 activity unit per microgram test protein. Silk fibroin at $350 \mu \mathrm{g}$ run with pepsin in SGF sampled at various time points. Lanes A-G contain samples of silk fibroin digested 
with pepsin at 0 (Lane A), 2 (Lane B), 5 (Lane C), 10 (Lane D), 20 (Lane E), 30 (Lane F), and 60 (Lane G) minute intervals.

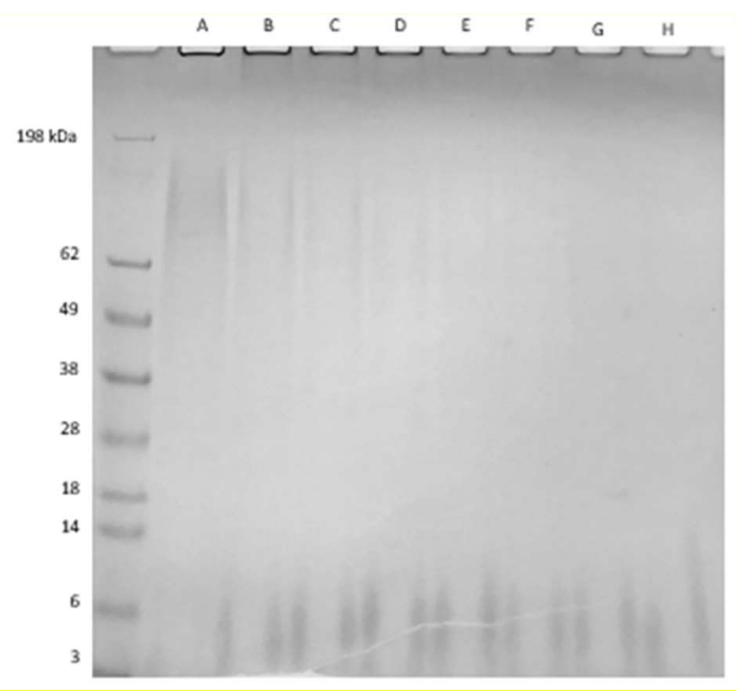

Figure 4. Reference Gel for the Digestion of Silk Fibroin in Pepsin Solution. To visualize the extent of silk fibroin digestion in pepsin solution, varying amounts $(\mu \mathrm{g})$ of silk fibroin were run SDS-PAGE gel to serve as a reference in the following quantities: $350 \mu \mathrm{g}(0 \%$ digestion, Lane A), $175 \mu \mathrm{g}$ (50\% digestion, Lane B), $88 \mu \mathrm{g}$ (75\% digestion, Lane C), $44 \mu \mathrm{g}$ (88\% digestion, Lane D), $22 \mu \mathrm{g}$ (93\% digestion, Lane E), $11 \mu \mathrm{g}$ (97\% digestion, Lane F), $5.5 \mu \mathrm{g}$ (98\% digestion, Lane $\mathrm{G}$ ), and $2.7 \mu \mathrm{g}$ (>99\% digestion, Lane $\mathrm{H})$.

\subsubsection{Identification \& Evaluation of Proteins of Allergenic Interest}

The following five proteins were identified as potentially allergenic components ("Proteins of Allergenic Interest") of silk cocoons and their derivatives: tropomyosin (TM), arginine kinase (AK), thioredoxin (TR), chitinase (CT), and paramyosin (PM). These were identified based on sequence homology to known allergens in similar arthropods or as a known protein in B. mori. Sequences corresponding to each protein of allergenic interest were identified by text search of protein name, and by BLAST searching to identify potentially unnamed members of the protein families.

Representative sequences from each Protein of Allergenic Interest were readily identifiable, at high confidence, in all three pupae samples (Table 8). This high-quality identification serves as a positive control, and suggests that the methodology employed is capable of definitive demonstration of protein presence if proteins are present in the sample. In some cases, isoforms of proteins of allergenic interest were identifiable due to the presence of peptides that are unique to one or another isoform. In other cases, only peptides that were shared between isoforms were identified, therefore positively identifying the group of proteins rather than an individual isoform. 
Table 8. Identification of proteins of allergenic interest, Score (Matches)

\begin{tabular}{llllll}
\hline & \multicolumn{1}{c}{ TM } & \multicolumn{1}{c}{ AK } & \multicolumn{1}{c}{ TR } & \multicolumn{1}{c}{ PM } \\
\hline Pupa1 & $237.67(7)$ & $318.42(16)$ & $227.14(9)$ & $348.47(13)$ & $159.95(5)$ \\
Pupa2a & $236.16(14)$ & $289.72(15)$ & $233.06(8)$ & $229.73(13)$ & $216.88(10)$ \\
Pupa2b & 225.47 (10) & $238.37(10)$ & $212.36(6)$ & $246.08(15)$ & $206.54(8)$ \\
Cocoon1 & nd (0) & nd (0) & 119.67(3) & nd (0) & nd (0) \\
Degummed SF 1 & nd (0) & nd (0) & nd (0) & nd (0) & nd (0) \\
SF Powder1 & nd (0) & nd (0) & nd (0) & nd (0) & nd (0) \\
SF Powder2a & nd (0) & nd (0) & nd (0) & nd (0) & nd (0) \\
SF Powder2b & 45.87 (1) & nd (0) & nd (0) & nd (0) & nd (0) \\
\hline
\end{tabular}

SF, silk fibroin; TM, tropomyosin; AK, arginine kinase; TR, thioredoxin; CN, chitinase; PM, paramyosin; nd, not detected

Score, -10logP score; Matches, number of peptide matches

For each protein of allergenic interest, the highest -10logP (ID quality) score obtained and the number of peptide matches (in parentheses) to that sequence is given.

One of three fibroin powder samples identified an equivocal match with one peptide from TM. However, the Human Proteome Organization (HUPO) requires at least two unique peptides for a protein identification. As such, this match does not represent a solid identity.

\subsubsection{Bioinformatics \& Literature Searches}

As confirmed by LC-MS/MS of fibroin, four proteins are dominant including fibroin heavy chain, Accession NP_001106733.1, which has 5263 amino acids (AA), and a mixture of fibroin light chain, Accession NP_001037488.1 which has 262 AA, fibroin P25 Accession CAA27804.1 with 220 AA and Sericin with Accession P07856.2 with 1186 AA. The full length sequences were compared to www.AllergenOnline.org version 20 using full-length FASTA, then a sliding 80 AA window that is adjusted for shorter segments to be equivalent to $80 \mathrm{AA}$, to search for identity matches greater than $35 \%$, and finally identity matches of 8 contiguous AA to allergens. In addition, these sequences were compared by BLASTP (version 2.10) to the NCBI Protein database to identify closest matches to proteins of any organism. The results for the primary proteins were evaluated with respect to similarity to known allergens and toxins and are summarized in Table 9.

Table 9. Bioinformatics search summary of identity matches (ID) for potential allergens in www.AllergenOnline.org with the dominant proteins in Fibroin, and by BLASTP to include allergens and toxins.

\begin{tabular}{|c|c|c|c|c|}
\hline Protein & FASTA3 overall & Sliding 80mer & 8 AA match & NCBI BLASTP 2.10 searches \\
\hline $\begin{array}{l}\text { Fibroin Heavy } \\
5263 \text { AA }\end{array}$ & $\begin{array}{l}\text { Collagen, } 26 \% \text { ID, } 1082 \\
\text { HMW gluten, } 25.7 \%, 654 \\
\text { AA } \\
\text { Gluten, } 26 \%, 710 \text { AA }\end{array}$ & $\begin{array}{l}\text { Ragweed Art v } 1 \\
\text { pollen, } 42.5 \text { to } 45 \% \text { ID } \\
\text { Parthenium pollen, } \\
41.3 \% \\
\text { Bovine collagen, } 38.2 \%\end{array}$ & None & $\begin{array}{l}\text { Limited by length, first } 402 \text { AA was } \\
\text { sufficient to search, matches were only } \\
\text { to lepidopteran sequences, none to } \\
\text { toxins or allergens }\end{array}$ \\
\hline $\begin{array}{l}\text { Fibroin light } 262 \\
\text { AA }\end{array}$ & $\begin{array}{l}\text { Globin CTT-IX, 30.8\%, } 78 \\
\text { AA }\end{array}$ & None & None & $\begin{array}{l}\text { Matches were only to lepidopteran } \\
\text { sequences, none to toxins or allergens }\end{array}$ \\
\hline
\end{tabular}


23 of 30

\begin{tabular}{|c|c|c|c|c|}
\hline & $\begin{array}{l}\text { SXP/RAL2, 24.8\%, } 153 \text { AA } \\
\text { SXP/RAL2, 24.8\%, } 153 \text { AA }\end{array}$ & & & \\
\hline $\begin{array}{l}\text { Fibroin P25 } \\
220 \text { AA }\end{array}$ & Sol I 1, 26.1\%, $115 \mathrm{AA}$ & None & None & $\begin{array}{l}\text { Matches were only to lepidopteran } \\
\text { sequences, none to toxins or allergens }\end{array}$ \\
\hline $\begin{array}{l}\text { Sericin } \\
1186 \mathrm{AA}\end{array}$ & $\begin{array}{l}\text { Gal g 6, } 27.9 \%, 330 \mathrm{AA} \\
\text { Disulfide isomerase, } 30.8 \% \text {, } \\
104 \mathrm{AA} \\
\text { Conglutin beta, } 18.8 \%, 415 \\
\text { AA }\end{array}$ & Gal g 6, 46.2\% & None & $\begin{array}{l}\text { Matches were only to lepidopteran } \\
\text { sequences, none to toxins or allergens }\end{array}$ \\
\hline
\end{tabular}

\section{Discussion}

In vitro and in vivo studies were conducted to assess the safety of silk fibroin, a naturally-derived material from the cocoons of Bombyx mori (silkworms) that has demonstrated promise as an edible coating to extend the shelf-life of foods. Silkworm cocoons are comprised largely of two primary protein components: sericin, which acts as a glue-like substance, and fibroin, which provides structural integrity. Using standard methods to isolate fibroin, sericin is removed by boiling cocoons under basic conditions [15]. The fibroin fibers are then dissolved in water using chaotropic salts. Subsequent removal of these salts by conventional methods results in solution that can then be applied to the surface of foods.

Silk fibroin was shown to be non-mutagenic in the bacterial reverse mutation (Ames) test. Five strains of bacteria and nine concentrations of silk fibroin were evaluated, up to a maximum dosage of 100,000 $\mathrm{gg}$ /plate. Similarly, silk fibroin did not demonstrate genotoxicity when evaluating micronuclei induction in mouse erythrocytes via flow cytometry. Here, mice were dosed with silk fibroin at 1,000 $\mathrm{mg} / \mathrm{kg}$ bodyweight/day, with blood samples collected for analysis 48 hours after treatment. A minimum of 4,000 polychromatic erythrocytes per animal were evaluated. Silk fibroin did not induce a statistically significant increase in frequency of micronucleated reticulocytes in male or female mice, nor did frequency of reticulocyte and micronucleated normochromatic erythrocytes differ significant after treatment with silk fibroin. Data from the above tests shows that silk fibroin is not genotoxic under the in vitro and in vivo conditions tested.

Chronic and acute systemic toxicity were evaluated in vivo with a 14-day repeat dose and rangefinding study followed by a 28-day repeat-dose oral consumption study in rats. During the 14-day study, rats were fed silk fibroin by oral gavage at doses between 0 and $500 \mathrm{mg} / \mathrm{kg} / \mathrm{day}$ (silk fibroin/bodyweight/day). No adverse clinical observations attributed to the administration of silk fibroin was observed. Due to the tolerance of the maximum dose of $500 \mathrm{mg} / \mathrm{kg} / \mathrm{day}$ in the 14-day feeding study, the same dose range was used in the 28-day study to assess systemic toxicity. As previously noted, the maximum dose could not be further increased due to test substance solubility and oral gavage volume constraints. Again, no silk fibroin-dependent effects were observed, including macroscopic and microscopic findings during necropsy after scheduled sacrifice and pathology.

Finally, an allergenicity risk assessment of silk fibroin was performed. Five potential allergens present in silkworms and their derivatives were identified. LC-MS/MS was used to determine whether these proteins were present in silk fibroin at various points during processing, including silkworm pupae, cocoons, and manufacturing intermediates. While all five proteins were easily detected in the silkworm pupae samples, they were not found in the cocoons or the silk fibroin sample that would be consumed as a food coating. Additionally, the test substance was rapidly digested by pepsin in SGF at a ratio of 10 units per microgram and moderately digested at a ratio of 1 unit per microgram. No degradation bands were found resulting from the digestion of silk fibroin. Based on CODEX 
(2003) guidelines for allergenicity assessment, there is no added concern of risk based on stability of silk fibroin in pepsin [19].

In order to assess potential allergenicity via cross-reactivity, the primary amino acid sequences present in silk fibroin were queried in the AllergenOnline database using FASTA3, which provides similar local alignments based on input query criteria. None of the full-length searches of silk fibroin resulted in $>50 \%$ identity. While 16 matches existed with $>35 \%$ identity over 80 amino acids using Entrez Protein BLASTP searches, the matches were low identity matches to proteins distinctly dissimilar to B. mori proteins. Silk fibroin therefore poses no concerns of allergenicity or potential cross-reactivity.

\section{Conclusions}

Silk fibroin has been shown to form an edible barrier on the surfaces of foods to extend shelf-life by reducing oxidation and moisture loss. This represents a transformative approach to food preservation that could have immense socioeconomic and environmental implications if widely implemented. Here, the safety of silk fibroin was evaluated using well-established in vivo, in vitro, and bioinformatics studies. First, a bacterial reverse mutation test (Ames test) conducted in five bacterial strains demonstrated that silk fibroin does not carry a mutagenic risk. Likewise, silk fibroin did not induce micronucleus formation in immature erythrocytes at the highest administered dose $(1,000$ $\mathrm{mg} / \mathrm{kg}$-bodyweight/day). Under silk fibroin solubility and oral gavage volume constraints, limited to $500 \mathrm{mg} / \mathrm{kg}$-bodyweight/day, a fourteen-day oral toxicity study in rats could not establish a maximum tolerable dose. A subsequent twenty-eight-day oral toxicity demonstrated no adverse macroscopic or microscopic clinical observations at the maximum dose. Next, an in vitro pepsin digestion assay was performed to assess the potential for silk fibroin to induce an allergenic response upon oral consumption. Here, silk fibroin did not demonstrate a high degree of stability and was rapidly digested by pepsin at ratio of 10 activity units per microgram test protein and intermediate stability at a ratio of 1 activity unit per microgram. Allergenic potential was further assessed by LC-MS/MS for detection of known allergenic arthropod proteins. Food allergy to consumption of silkworm pupae has been described and is likely due to five known allergenic proteins. The five allergenic proteins were readily detected in silkworm pupae but were not found in the cocoon before or after processing, nor in the final silk fibroin product. Finally, bioinformatics analysis did not identify any matches that would suggest significant identity matches to allergens or toxins. The results of the studies presented in this article raise no questions of toxicological or allergenic concerns at doses up to and including $500 \mathrm{mg} / \mathrm{kg}$ bodyweight per day. 


\section{Supplementary Materials:}

Table S1: Historical control data: Mean revertants per plate \pm SD (Ames test), Table S2. Validation data: cyclophosphamide, Table S3. Historical control data (micronucleated reticulocytes), Figure S1A Pepsin Digestion of bovine serum albumin (BSA) at a ratio of 10 activity units per microgram test protein, Figure S1B Pepsin Digestion of bovine serum albumin (BSA) at a ratio of 1 activity unit per microgram test protein, Figure S2 Pepsin in SGF sampled for stability.

Bacterial Reverse Mutation Test (Ames Test)

Table S1. Historical control data: Mean revertants per plate \pm SD (Ames test $)^{1}$

\begin{tabular}{|c|c|c|c|c|c|c|c|}
\hline \multirow[b]{2}{*}{ Strain } & \multirow[b]{2}{*}{ Treatment } & \multirow[b]{2}{*}{ Dose } & \multirow[b]{2}{*}{ S9 } & \multicolumn{2}{|c|}{ Plate Incorporation Method } & \multicolumn{2}{|c|}{$\begin{array}{l}\text { Pre-Incubation } \\
\text { Method }\end{array}$} \\
\hline & & & & Mean \pm SD & Range & Mean \pm SD & Range \\
\hline TA1535 & Sodium azide & 1.5 & - & $618 \pm 91$ & $359-1192$ & $622 \pm 71$ & $478-831$ \\
\hline TA1537 & ICR 191 Acridine & 1 & - & $1136 \pm 1437$ & $119-6388$ & $3227 \pm 1227$ & $875-5700$ \\
\hline TA98 & Daunomycin & 6 & - & $938 \pm 343$ & $350-1500$ & $602 \pm 345$ & $146-1227$ \\
\hline TA100 & Sodium azide & 1.5 & - & $600 \pm 126$ & $394-1003$ & $539 \pm 166$ & $138-904$ \\
\hline E. Coli & MMS & 2.5 & - & $634 \pm 101$ & $389-846$ & $509 \pm 143$ & $313-808$ \\
\hline TA1535 & $2-\mathrm{AA}$ & 10 & + & $267 \pm 86$ & $85-636$ & $293 \pm 64$ & $64-391$ \\
\hline TA1537 & $2-\mathrm{AA}$ & 10 & + & $280 \pm 99$ & $42-512$ & $260 \pm 107$ & $112-541$ \\
\hline TA98 & $2-\mathrm{AA}$ & 10 & + & $2321 \pm 971$ & $83-3915$ & $2384 \pm 938$ & $506-3530$ \\
\hline TA100 & $2-\mathrm{AA}$ & 10 & + & $2377 \pm 806$ & $976-4169$ & $2388 \pm 583$ & $1308-3620$ \\
\hline E. Coli & $2-\mathrm{AA}$ & 10 & + & $125 \pm 30$ & $63-196$ & $128 \pm 29$ & $60-188$ \\
\hline TA1535 & Sterile Water & $\mathrm{N} / \mathrm{A}$ & - & $13 \pm 2$ & $7-21$ & $16 \pm 3$ & $8-23$ \\
\hline TA1537 & Sterile Water & $\mathrm{N} / \mathrm{A}$ & - & $12 \pm 4$ & $6-25$ & $14 \pm 5$ & $5-23$ \\
\hline TA98 & Sterile Water & $\mathrm{N} / \mathrm{A}$ & - & $28 \pm 8$ & $16-49$ & $29 \pm 7$ & $14-46$ \\
\hline TA100 & Sterile Water & $\mathrm{N} / \mathrm{A}$ & - & $130 \pm 16$ & $104-155$ & $118 \pm 18$ & $83-143$ \\
\hline E. Coli & Sterile Water & $\mathrm{N} / \mathrm{A}$ & - & $45 \pm 7$ & $29-57$ & $46 \pm 10$ & $30-67$ \\
\hline TA1535 & Sterile Water & $\mathrm{N} / \mathrm{A}$ & + & $13 \pm 1$ & $9-20$ & $12 \pm 2$ & $8-19$ \\
\hline TA1537 & Sterile Water & $\mathrm{N} / \mathrm{A}$ & + & $15 \pm 3$ & $8-28$ & $14 \pm 5$ & $6-26$ \\
\hline TA98 & Sterile Water & $\mathrm{N} / \mathrm{A}$ & + & $29 \pm 5$ & $18-40$ & $35 \pm 7$ & $23-50$ \\
\hline TA100 & Sterile Water & $\mathrm{N} / \mathrm{A}$ & + & $145 \pm 14$ & $116-170$ & $125 \pm 16$ & $88-147$ \\
\hline E. Coli & Sterile Water & N/A & + & $59 \pm 13$ & $13-31$ & $53 \pm 9$ & $36-76$ \\
\hline
\end{tabular}

${ }^{1}$ Historical Data maintained by PSL from 2015

$\mathrm{SD}$, standard deviation; dose units $\mu \mathrm{g} /$ plate; range is $\min -\max$

In Vivo Mouse Erythrocyte Micronucleus Test (Flow Cytometry)

Table S2. Validation data: cyclophosphamide (from PSL)

\begin{tabular}{lcc}
\hline$\%$ MN-RET & \multicolumn{2}{c}{ Mean \pm SEM } \\
\hline Treatment & $M$ & $\mathrm{~F}$ \\
\hline $0 \mathrm{mg} / \mathrm{kg}$ & $0.17 \pm 0.02$ & $0.17 \pm 0.02$ \\
$1.5 \mathrm{mg} / \mathrm{kg}$ & $0.23 \pm 0.03$ & $0.22 \pm 0.04$ \\
$5 \mathrm{mg} / \mathrm{kg}$ & $0.28^{\mathrm{a}} \pm 0.03$ & $0.29^{\mathrm{a}} \pm 0.02$
\end{tabular}




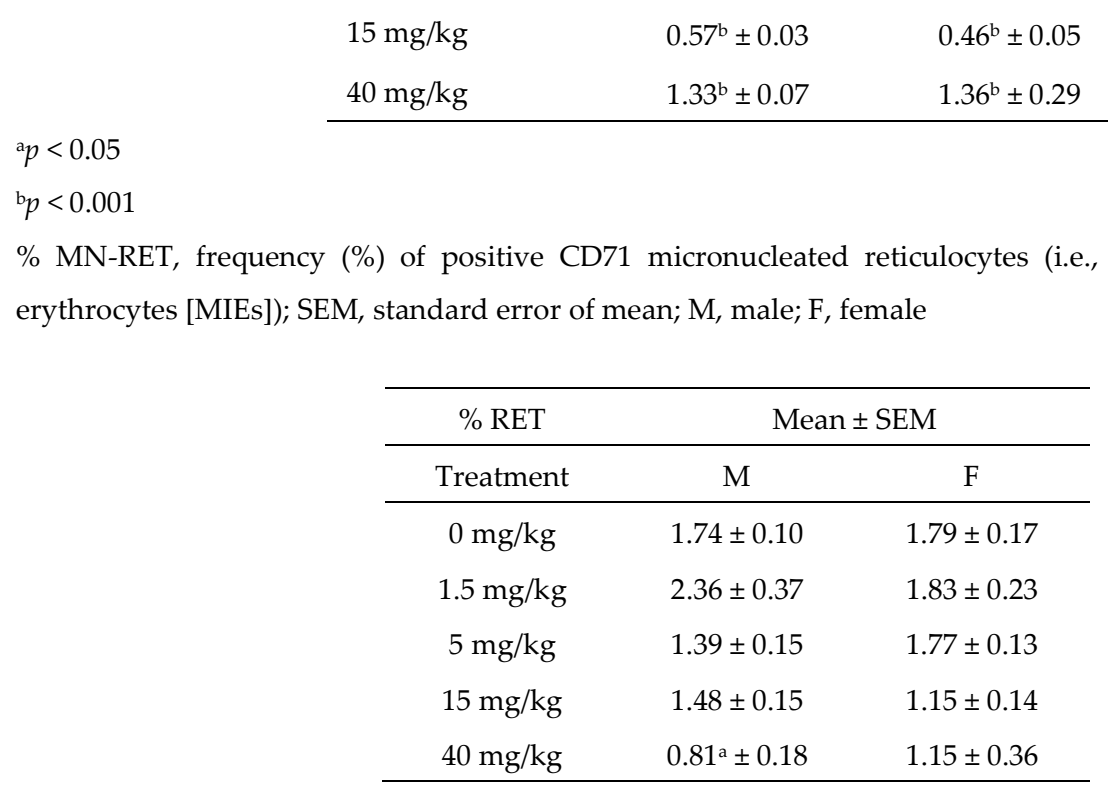

a $p<0.05$

$\%$ RET, frequency (\%) of CD71 positive reticulocytes; SEM, standard error of mean; M, male; F, female

\begin{tabular}{ccc}
\hline$\%$ MN-NCE & \multicolumn{2}{c}{ Mean \pm SEM } \\
\hline Treatment & M & F \\
\hline $0 \mathrm{mg} / \mathrm{kg}$ & $0.12 \pm 0.01$ & $0.11 \pm 0.01$ \\
$1.5 \mathrm{mg} / \mathrm{kg}$ & $0.11 \pm 0.02$ & $0.11 \pm 0.01$ \\
$5 \mathrm{mg} / \mathrm{kg}$ & $0.10 \pm 0.01$ & $0.10 \pm 0.01$ \\
$15 \mathrm{mg} / \mathrm{kg}$ & $0.15 \pm 0.00$ & $0.11 \pm 0.01$ \\
$40 \mathrm{mg} / \mathrm{kg}$ & $0.12 \pm 0.01$ & $0.14 \pm 0.02$ \\
\hline
\end{tabular}

$\%$ MN-NCE, frequency (\%) of micronucleated normochromatic erythrocytes; SEM, standard error of mean; $\mathrm{M}$, male; F, female

Table S3. Historical control data (from PSL).

\begin{tabular}{lcccc}
\hline$\%$ MN-RET & \multicolumn{2}{c}{ Mean \pm SEM } & \multicolumn{2}{c}{ Control limits } \\
\hline Treatment & M & F & M & F \\
\hline Vehicle & $0.16 \pm 0.01$ & $0.17 \pm 0.01$ & $-0.01-0.34$ & $-0.07-0.41$ \\
CP 40 & $1.56 \pm 0.08$ & $1.49 \pm 0.12$ & $-0.12-3.24$ & $-1.04-4.02$ \\
\hline
\end{tabular}

$\%$ MN-RET, frequency (\%) of positive CD71 micronucleated reticulocytes (i.e., micronucleated immature erythrocytes [MIEs]); M, male; F, female

\begin{tabular}{lcccc}
\hline$\%$ RET & \multicolumn{2}{c}{ Mean \pm SEM } & \multicolumn{2}{c}{ Control limits } \\
\hline Treatment & $\mathrm{M}$ & $\mathrm{F}$ & $\mathrm{M}$ & $\mathrm{F}$ \\
\hline Vehicle & $2.21 \pm 0.15$ & $1.90 \pm 0.08$ & $-0.80-5.22$ & $0.27-3.54$ \\
CP 40 & $0.69 \pm 0.05$ & $0.81 \pm 0.06$ & $-0.29-1.68$ & $-0.54-2.17$ \\
\hline
\end{tabular}

$\%$ RET, frequency (\%) of CD71 positive reticulocytes; M, male; F, female 


\begin{tabular}{lcccc}
\hline$\%$ MN-NCE & \multicolumn{2}{c}{ Mean \pm SEM } & \multicolumn{2}{c}{ Control limits } \\
\hline Treatment & M & F & M & F \\
\hline Vehicle & $0.12 \pm 0.00$ & $0.11 \pm 0.01$ & $0.03-0.22$ & $-0.04-0.26$ \\
CP 40 & $0.12 \pm 0.00$ & $0.13 \pm 0.00$ & $0.02-0.23$ & $0.04-0.22$ \\
\hline
\end{tabular}

$\%$ MN-NCE, frequency (\%) of micronucleated normochromatic erythrocytes; M, male; F, female

Pepsin Digestion

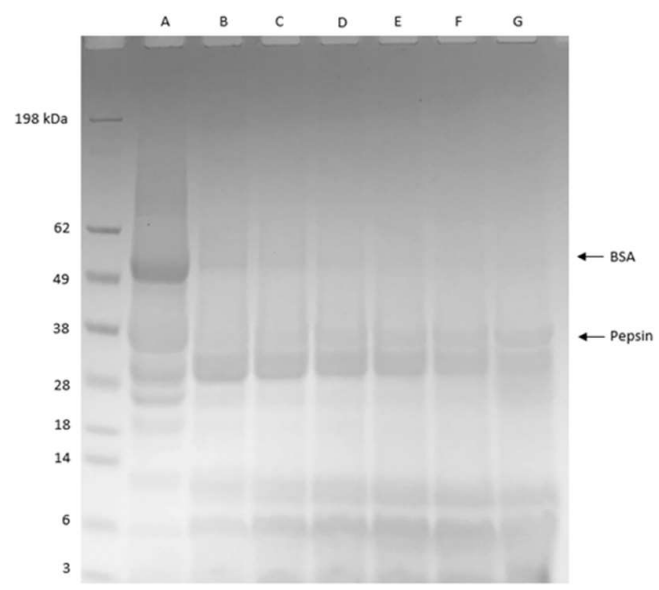

Figure S1A Pepsin Digestion of bovine serum albumin (BSA) at a ratio of 10 activity units per microgram test protein. Silk fibroin at $350 \mu \mathrm{g}$ run with pepsin in SGF sampled at various time points. Lanes A-G contain samples of silk fibroin digested with pepsin at 0 (Lane A), 2 (Lane B), 5 (Lane C), 10 (Lane D), 20 (Lane E), 30 (Lane F), and 60 (Lane G) minute intervals.

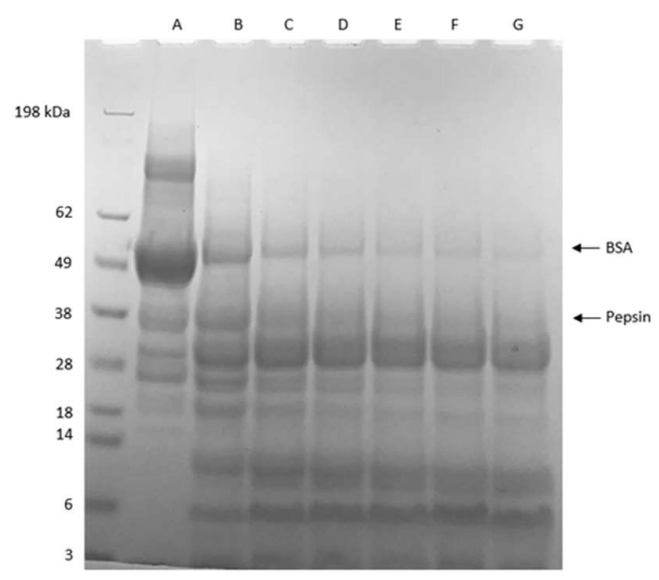

Figure S1B Pepsin Digestion of bovine serum albumin (BSA) at a ratio of 1 activity unit per microgram test protein. Silk fibroin at $350 \mu \mathrm{g}$ run with pepsin in SGF sampled at various time points. Lanes A-G contain samples of silk fibroin digested with pepsin at 0 (Lane A), 2 (Lane B), 5 (Lane C), 10 (Lane D), 20 (Lane E), 30 (Lane F), and 60 (Lane G) minute intervals. 


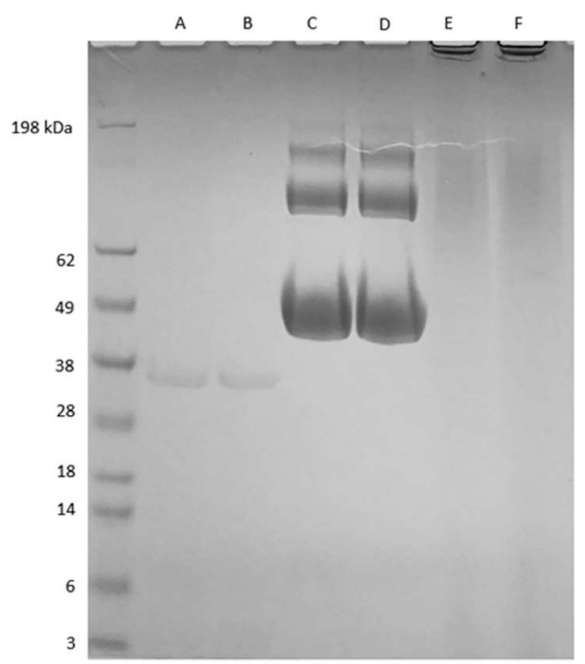

Figure S2 Pepsin in SGF sampled for stability at $0 \mathrm{~min}$. (Lane A) and $60 \mathrm{~min}$. (Lane B). BSA in SGF sampled for stability at $0 \mathrm{~min}$. (Lane C) and $60 \mathrm{~min}$. (Lane D). Silk fibroin in SGF sampled for stability at $0 \mathrm{~min}$. (Lane E) and 60 min. (Lane F).

Author Contributions: Conceptualization, Sezin Yigit, Nadia Hallaj, James Sugarman, Laith Abu-Taleb, Richard Goodman, Philip Johnson and Adam Behrens; Data curation, Sezin Yigit, Nadia Hallaj, James Sugarman, Samantha Roman, Laith Abu-Taleb, Richard Goodman, Philip Johnson and Adam Behrens; Formal analysis, Sezin Yigit, Nadia Hallaj, James Sugarman, Lester Chong, Samantha Roman, Laith Abu-Taleb, Richard Goodman, Philip Johnson and Adam Behrens; Investigation, Sezin Yigit, Nadia Hallaj, James Sugarman, Lester Chong, Laith Abu-Taleb, Richard Goodman, Philip Johnson and Adam Behrens; Methodology, Sezin Yigit, Nadia Hallaj, James Sugarman, Laith Abu-Taleb, Richard Goodman, Philip Johnson and Adam Behrens; Project administration, Sezin Yigit, Laith Abu-Taleb, Richard Goodman, Philip Johnson and Adam Behrens; Resources, Nadia Hallaj, Laith Abu-Taleb, Richard Goodman, Philip Johnson and Adam Behrens; Supervision, Sezin Yigit, Laith Abu-Taleb, Richard Goodman, Philip Johnson and Adam Behrens; Validation, Nadia Hallaj, James Sugarman, Lester Chong, Samantha Roman, Laith Abu-Taleb, Richard Goodman, Philip Johnson and Adam Behrens; Visualization, Sezin Yigit, Nadia Hallaj, James Sugarman, Samantha Roman, Richard Goodman, Philip Johnson and Adam Behrens; Writing - original draft, Nadia Hallaj, Lester Chong, Samantha Roman, Laith AbuTaleb and Adam Behrens; Writing - review \& editing, Nadia Hallaj, James Sugarman, Laith Abu-Taleb, Richard Goodman, Philip Johnson and Adam Behrens. All authors have read and agreed to the published version of the manuscript.

Funding: This research received no additional funding other than that described in the Conflicts of Interest Statement.

Acknowledgments: The authors would like to thank Product Safety Laboratories, Inc. (Dayton, New Jersey) for conducting our bacterial reverse mutation test (Ames test), our 14-day range finder study, and 28-day oral toxicity study. In particular, we thank Raghavendra Gowda at PSL.

Conflicts of Interest: S. Yigit, N. Hallaj, J. Sugarman, L. Chong, S. Roman, L. Abu-Taleb, and A. Behrens are employees of Cambridge Crops, Inc. (d.b.a. Mori), the developer of the silk fibroin product. R. Goodman and P. Johnson have received consulting fees for work with Cambridge Crops and for recommendations on food safety evaluation procedures. The studies described in this article were paid for by Cambridge Crops. 


\section{References}

1. Marelli, B.; Brenckle, M. A.; Kaplan, D. L.; Omenetto, F. G. Silk Fibroin as Edible Coating for Perishable Food Preservation. Scientific Reports 2016, 6 (1).

2. Behrens A., Yigit, S., Abu-Taleb, L., Hallaj, N., Preston, C., Roman, S., Sugarman, J. Compositions and Methods for Polymer Based Shelf Life Extension. US 2020/0178576 Al.

3. FAO. 2011. Global food losses and food waste - Extent, causes, and prevention. Rome.

4. Jayas, D.; Jeyamkondan, S. PH-Postharvest Technology. Biosystems Engineering 2002, 82 (3), $235-251$.

5. Li, L.; Zhang, M.; Adhikari, B.; Gao, Z. Recent Advances in Pressure Modification-Based Preservation Technologies Applied to Fresh Fruits and Vegetables. Food Reviews International 2016, 33 (5), 538-559.

6. Tzortzakis, N.; Chrysargyris, A. Postharvest Ozone Application for the Preservation of Fruits and Vegetables. Food Reviews International 2016, 33 (3), 270-315.

7. Environmental Protection Agency, 2019. Advancing Sustainable Materials Management: 2017 Fact Sheet. Assessing Trends in Material Generation, Recycling, Composting, Combustion with Energy Recovery and Landfilling in the United States. https://www.epa.gov/sites/production/files/201911/documents/2017_facts_and_figures_fact_sheet_final.pdf (accessed February 19, 2020).

8. Dilkes-Hoffman, L. S.; Lane, J. L.; Grant, T.; Pratt, S.; Lant, P. A.; Laycock, B. Environmental Impact of Biodegradable Food Packaging When Considering Food Waste. Journal of Cleaner Production 2018, 180, 325-334.

9. Khan, B.; Niazi, M. B. K.; Samin, G.; Jahan, Z. Thermoplastic Starch: A Possible Biodegradable Food Packaging Material-A Review. Journal of Food Process Engineering 2016, 40 (3).

10. Marcos, B.; Aymerich, T.; Monfort, J. M.; Garriga, M. Physical Performance of Biodegradable Films Intended for Antimicrobial Food Packaging. Journal of Food Science 2010, 75 (8).

11. Scarfato, P.; Maio, L. D.; Incarnato, L. Recent Advances and Migration Issues in Biodegradable Polymers from Renewable Sources for Food Packaging. Journal of Applied Polymer Science 2015, 132 (48).

12. Savithri G.; Sujathamma P. Mulberry and Silkworm As A Healthy Foodstuff - A Review. Int J Recent Sci Res. 2016, 7 (6), 12244-12246.

13. Rajakumar, S.; Bindroo, B.B. Food and Medicinal Values in Silkworm and its Host Plant Mulberry. Int J Food Nut Sci. 2014, 3 (1).

14. Ramos-Elorduy, J. Anthropo-entomophagy: Cultures, evolution, and sustainability. Entomological Research 2009, 3 (9), 271-288.

15. Heo HS, Choi JH, Oh JJ, Lee WJ, Kim SS, Lee DH, Lee HK, Song SW, Kim KH, Choi YK, Ryu KS. Evaluation of general toxicity and genotoxicity of the silkworm extract powder. Toxicological research. 2013, 29 (4):26378.

16. US FDA Toxicological Principles for the Safety Assessment of Food Ingredients, Redbook 2000, Revised 2018 IV.C.1.A. Bacterial Reverse Mutation Test (2003).

17. Test No. 407: Repeated Dose 28-Day Oral Toxicity Study in Rodents. OECD Guidelines for the Testing of Chemicals, Section 42008.

18. US FDA Toxicological Principles for the Safety Assessment of Food Ingredients, Redbook 2000, Revised 2007 IV.C. 4. A. Subchronic Toxicity Studies with Rodents (2003).

19. Codex Alimentarius Commission. Alinorm 03/34: Joint FAO/WHO Food Standard Programme, Codex Alimentarius Commission, Twenty-Fifth Session, Rome, Italy 30 June-5 July, 2003. Appendix III, Guideline for the conduct of food safety assessment of foods derived from recombinant-DNA plants and Appendix IV, Annex on the assessment of possible allergenicity. 2003;4760.

20. Ofori-Anti, A.; Ariyarathna, H.; Chen, L.; Lee, H.; Pramod, S.; Goodman, R. Establishing Objective Detection Limits for the Pepsin Digestion Assay Used in the Assessment of Genetically Modified Foods. Regulatory Toxicology and Pharmacology 2008, 52 (2), 94-103.

21. Thomas, K.; Aalbers, M.; Bannon, G.; Bartels, M.; Dearman, R.; Esdaile, D.; Fu, T.; Glatt, C.; Hadfield, N.; Hatzos, C.; Hefle, S.; Heylings, J.; Goodman, R.; Henry, B.; Herouet, C.; Holsapple, M.; Ladics, G.; Landry, T.; Macintosh, S.; Rice, E.; Privalle, L.; Steiner, H.; Teshima, R.; Ree, R. V.; Woolhiser, M.; Zawodny, J. A Multi-Laboratory Evaluation of a Common in Vitro Pepsin Digestion Assay Protocol Used in Assessing the Safety of Novel Proteins. Regulatory Toxicology and Pharmacology 2004, 39 (2), 87-98.

22. Rockwood, D. N.; Preda, R. C.; Yücel, T.; Wang, X.; Lovett, M. L.; Kaplan, D. L. Materials Fabrication from Bombyx Mori Silk Fibroin. Nature Protocols 2011, 6 (10), 1612-1631. 
23. Test No. 471: Bacterial Reverse Mutation Test. OECD Guidelines for the Testing of Chemicals, Section 4 2020.

24. Maron, D. M.; Ames, B. N. Revised Methods for the Salmonella Mutagenicity Test. Mutation Research/Environmental Mutagenesis and Related Subjects 1983, 113 (3-4), 173-215.

25. Mortelmans, K.; Zeiger, E. The Ames Salmonella/Microsome Mutagenicity Assay. Mutation Research/Fundamental and Molecular Mechanisms of Mutagenesis 2000, 455 (1-2), 29-60.

26. Gatehouse, D. Bacterial Mutagenicity Assays: Test Methods. Methods in Molecular Biology Genetic Toxicology 2011, 21-34.

27. Test No. 474: Mammalian Erythrocyte Micronucleus Test. OECD Guidelines for the Testing of Chemicals, Section 42016.

28. National Research Council (U.S.) Committee for the Update of the Guide for the Care and Use of Laboratory Animals. 2011. Guide for the Care and Use of Laboratory Animals. 8th ed. National Academies Press (U.S.), Washington.

29. U.S. EPA Health Effects Test Guidelines, 2009. OPPTS 870.3050 Repeated Dose 28-day Oral Toxicity in Rodents. https://www.regulations.gov/document?D=EPA-HQ-OPPT-2009-0156-0009 (accessed 31 January 2020).

30. U.S. Department of Health and Human Services, Food and Drug Administration, Center for Food Safety and Applied Nutrition, 2003. Chapter IV.C.4.a. Subchronic Toxicity Studies with Rodents. Guidance for Industry and Other Stakeholders Toxicological Principles for the Safety Assessment of Food Ingredients Redbook 2000. https://www.fda.gov/regulatory-information/search-fda-guidance-documents/redbook2000-ivc1a-bacterial-reverse-mutation-test (accessed 31 January 2020).

31. Jin, Y.; Goodman, R. E.; Tetteh, A. O.; Lu, M.; Tripathi, L. Bioinformatics Analysis to Assess Potential Risks of Allergenicity and Toxicity of HRAP and PFLP Proteins in Genetically Modified Bananas Resistant to Xanthomonas Wilt Disease. Food and Chemical Toxicology 2017, 109, 81-89.

32. Boeck, M. D.; Leede, B.-J. V. D.; Goethem, F. V.; Smedt, A. D.; Steemans, M.; Lampo, A.; Vanparys, P. Flow Cytometric Analysis of Micronucleated Reticulocytes: Time- and Dose-Dependent Response of Known Mutagens in Mice, Using Multiple Blood Sampling. Environmental and Molecular Mutagenesis 2005, 46 (1), 30-42.

33. Lebaron, M. J.; Schisler, M. R.; Torous, D. K.; Dertinger, S. D.; Gollapudi, B. B. Influence of Counting Methodology on Erythrocyte Ratios in the Mouse Micronucleus Test. Environmental and Molecular Mutagenesis 2012, 54 (3), 222-228.

34. Witt, K. L.; Livanos, E.; Kissling, G. E.; Torous, D. K.; Caspary, W.; Tice, R. R.; Recio, L. Comparison of Flow Cytometry- and Microscopy-Based Methods for Measuring Micronucleated Reticulocyte Frequencies in Rodents Treated with Nongenotoxic and Genotoxic Chemicals. Mutation Research/Genetic Toxicology and Environmental Mutagenesis 2008, 649 (1-2), 101-113. 